\title{
Positive-breakdown regression by minimizing nested scale estimators
}

\author{
Christophe Croux ${ }^{\dagger}$, Peter J. Rousseeuw, and Annemie Van Bael \\ Universitaire Instelling Antwerpen, Belgium
}

Abstract: In this article we present a new class of robust regression estimators. Our main example is the least trimmed median estimator (LTM). It minimizes the objective function $\sum_{k=1}^{h_{p}}\left\{\operatorname{median}_{j}\left|r_{i}(\boldsymbol{\beta})-r_{j}(\boldsymbol{\beta})\right|\right\}_{(k)}$ where $h_{p}=[(n+p+1) / 2]$ and the subscript $(k)$ indicates the k-th order statistic. It can be seen as an alternative to the least median of squares (LMS) and the least trimmed squares (LTS) estimators, which minimize the objective functions $|r|_{\left(h_{p}\right)}$ and $\sum_{k=1}^{h_{p}} r_{(k)}^{2}$. The LTM is not geared towards symmetric error distributions, which makes it more generally applicable. We will see that the LTM has the same breakdown point as the LMS and the LTS, but that its gaussian efficiency is higher. We will also show that the LTM has a much better bias curve than that of the LTS (derived in this paper), and that its computation time is virtually the same. The LTM is illustrated on a real data set about concentrations of plutonium isotopes. We also compare the LTM to various other estimators, including S-estimators and one-step versions of Mallows and Schweppe estimators.

AMS Subject Classification: 62F35, 62J05.

Key words and phrases: Bias curve; breakdown point; efficiency; influence function.

Correspondence to: C. Croux, Department of Mathematics and Computer Science, Universitaire Instelling Antwerpen (U.I.A.), Universiteitsplein 1, B-2610 Antwerp, Belgium.

\footnotetext{
${ }^{\dagger}$ Christophe Croux is Research Assistant with the Belgian National Fund for Scientific Research (NFWO).
} 


\section{INTRODUCTION}

The linear model

$$
y_{i}=\boldsymbol{\beta}^{t} \mathbf{x}_{i}+\alpha+\sigma \varepsilon_{i} \quad \text { for } i=1, \ldots, n
$$

contains a slope parameter $\boldsymbol{\beta}$, an intercept term $\alpha$ and a scale parameter $\sigma$. The carriers $\mathbf{x}_{i}$ are (p-1)-dimensional vectors. We assume that the errors $\varepsilon_{i}$ are independent and identically distributed according to a distribution $F$, and that the $\varepsilon_{i}$ are independent of the $\mathbf{x}_{i}$ which follow a distribution $G$. We will denote residuals as $r_{i}(\boldsymbol{\beta}, \alpha)=y_{i}-\boldsymbol{\beta}^{t} \mathbf{x}_{i}-\alpha$ for any $\boldsymbol{\beta}$ and $\alpha$. We also define the quantities $r_{i}(\boldsymbol{\beta}):=y_{i}-\boldsymbol{\beta}^{t} \mathbf{x}_{i}$ which do not depend on $\alpha$.

The classical least squares (LS) estimator for the regression parameters is defined as

$$
\left(\hat{\boldsymbol{\beta}}_{\mathrm{LS}}, \hat{\alpha}_{\mathrm{LS}}\right)=\underset{(\boldsymbol{\beta}, \alpha)}{\operatorname{argmin}}\left(\frac{1}{n} \sum_{i=1}^{n} r_{i}(\boldsymbol{\beta}, \alpha)^{2}\right)^{1 / 2} .
$$

We can rewrite this to estimate the slope separately:

$$
\hat{\boldsymbol{\beta}}_{\mathrm{LS}}=\underset{\boldsymbol{\beta}}{\operatorname{argmin}}\left(\frac{1}{n} \sum_{i=1}^{n}\left(r_{i}(\boldsymbol{\beta})-t_{\mathrm{LS}}\left(r_{1}(\boldsymbol{\beta}), \ldots, r_{n}(\boldsymbol{\beta})\right)\right)^{2}\right)^{1 / 2}
$$

where the univariate location estimator $t_{\mathrm{LS}}$ is given by

$$
t_{\mathrm{LS}}\left(r_{1}(\boldsymbol{\beta}), \ldots, r_{n}(\boldsymbol{\beta})\right)=\frac{1}{n} \sum_{i=1}^{n} r_{i}(\boldsymbol{\beta})=\underset{\alpha}{\operatorname{argmin}}\left(\frac{1}{n} \sum_{i=1}^{n}\left(r_{i}(\boldsymbol{\beta})-\alpha\right)^{2}\right)^{1 / 2}
$$

This means that (1.1) estimates the slope by the vector $\hat{\boldsymbol{\beta}}$ which minimizes the standard deviation of the $r_{i}(\boldsymbol{\beta})$. Once $\hat{\boldsymbol{\beta}}$ is computed we can then put $\hat{\alpha}:=t_{\mathrm{LS}}\left(r_{1}(\hat{\boldsymbol{\beta}}), \ldots, r_{n}(\hat{\boldsymbol{\beta}})\right)$.

It is well-known that the least squares estimator is very sensitive to outliers. By contrast, the least median of squares (LMS) estimator (Rousseeuw 1984) can resist outliers in the response variable as well as in the regressors. It is defined as

$$
\left(\hat{\boldsymbol{\beta}}_{\mathrm{LMS}}, \hat{\alpha}_{\mathrm{LMS}}\right)=\underset{(\boldsymbol{\beta}, \alpha)}{\operatorname{argmin}}\left\{\left|r_{i}(\boldsymbol{\beta}, \alpha)\right| ; i=1, \ldots, n\right\}_{\left(h_{p}\right)}
$$

where $h_{p}=[(n+p+1) / 2]$. (The subscript $(k)$ indicates the $\mathrm{k}$-th order statistic out of the indicated set.) Also here, we can estimate the slope separately:

$$
\hat{\boldsymbol{\beta}}_{\mathrm{LMS}}=\underset{\boldsymbol{\beta}}{\operatorname{argmin}}\left\{\left|r_{i}(\boldsymbol{\beta})-t_{\mathrm{LMS}}\left(r_{1}(\boldsymbol{\beta}), \ldots, r_{n}(\boldsymbol{\beta})\right)\right| ; i=1, \ldots, n\right\}_{\left(h_{p}\right)}
$$

where the univariate location estimator

$$
t_{\mathrm{LMS}}\left(r_{1}(\boldsymbol{\beta}), \ldots, r_{n}(\boldsymbol{\beta})\right)=\underset{\alpha}{\operatorname{argmin}}\left\{\left|r_{i}(\boldsymbol{\beta})-\alpha\right| ; i=1, \ldots, n\right\}_{\left(h_{p}\right)}
$$


can be computed very quickly (Rousseeuw and Leroy 1987, page 169). The objective function (1.3) can be seen as a robustification of (1.1). Unfortunately, $\hat{\boldsymbol{\beta}}_{\text {LMS }}$ has a non-standard convergence. Therefore, Rousseeuw (1983) introduced the least trimmed squares (LTS) estimator, which is given by

$$
\hat{\boldsymbol{\beta}}_{\mathrm{LTS}}=\underset{\boldsymbol{\beta}}{\operatorname{argmin}}\left(\frac{1}{h_{p}} \sum_{k=1}^{h_{p}}\left\{\left(r_{i}(\boldsymbol{\beta})-t_{\mathrm{LTS}}\left(r_{1}(\boldsymbol{\beta}), \ldots, r_{n}(\boldsymbol{\beta})\right)\right)^{2} ; i=1, \ldots, n\right\}_{(k)}\right)^{1 / 2}
$$

where the location estimator

$$
t_{\mathrm{LTS}}\left(r_{1}(\boldsymbol{\beta}), \ldots, r_{n}(\boldsymbol{\beta})\right)=\underset{\alpha}{\operatorname{argmin}}\left(\frac{1}{h_{p}} \sum_{k=1}^{h_{p}}\left\{\left(r_{i}(\boldsymbol{\beta})-\alpha\right)^{2} ; i=1, \ldots, n\right\}_{(k)}\right)^{1 / 2}
$$

can be computed as in (Rousseeuw and Leroy 1987, page 171). The LTS slope estimator is asymptotically normal, but its efficiency is very low. Therefore we propose a new estimator, which we will call the least trimmed median (LTM). It is defined by

$$
\hat{\boldsymbol{\beta}}_{\mathrm{LTM}}=\underset{\boldsymbol{\beta}}{\operatorname{argmin}} \frac{1}{h_{p}} \sum_{k=1}^{h_{p}}\left\{\underset{j}{\operatorname{median}}\left|r_{i}(\boldsymbol{\beta})-r_{j}(\boldsymbol{\beta})\right|\right\}_{(k)}
$$

where this median stands for the $([n / 2]+1)$-th order statistic out of $n$ numbers, and is often referred to as the "high median". (Equivalently, we may take the "low median" over all $j \neq i$.) For a given value of $\boldsymbol{\beta}$, we compute the objective function as follows: for each $r_{i}(\boldsymbol{\beta})$ we compute $\operatorname{median}_{j}\left|r_{i}(\boldsymbol{\beta})-r_{j}(\boldsymbol{\beta})\right|$. This yields $n$ medians. Computing the average of the smallest $h_{p}$ of these medians yields the objective function.

All four slope estimators considered above can thus be written as

$$
\hat{\boldsymbol{\beta}}=\underset{\boldsymbol{\beta}}{\operatorname{argmin}} s_{n}\left(r_{1}(\boldsymbol{\beta}), \ldots, r_{n}(\boldsymbol{\beta})\right)
$$

where the objective function $s_{n}$ is a scale estimator which is shift invariant, and hence does not depend on $\alpha$. Note, however, that this shift invariance is obtained in different ways. In the objective functions of LS, LMS, and LTS, the $r_{i}(\boldsymbol{\beta})$ are first centered by subtracting a location estimate $t_{n}\left(r_{1}, \ldots, r_{n}\right)$. (This amounts to an "intercept adjustment" at each value of the slope parameter $\boldsymbol{\beta}$.) These scale estimators are location-based in the sense that they depend on the choice of the initial location estimator. Also, they take a symmetric view on dispersion, because all three scale estimators depend only on the absolute deviations $\left|r_{i}-t_{n}\right|$ from the central location $t_{n}$, hence positive deviations are attached exactly the same 
importance as negative deviations. This is because the LS, LMS, and LTS were intended primarily for models with a symmetric error distribution.

By contrast, the objective function of the LTM does not contain any location estimator, because it depends only on the pairwise distances $\left|r_{i}-r_{j}\right|$. This location-free scale estimator was proposed by Rousseeuw and Croux $(1992,1993)$. By construction, it is not restricted to symmetric distributions. Therefore, the LTM slope estimator can also be used for asymmetric error distributions. Moreover, it can estimate the slope consistently without regard to the intercept, like the slope estimators of Theil (1950) and Sen (1968) in simple regression. Note that some robust slope estimators (notably, GM estimators of Schweppe type) become inconsistent at asymmetric error distributions (Carroll and Welsh 1988).

For slope estimators of the type (1.8) we can estimate the parameter $\sigma$ by the minimal value of the objective function that has been attained:

$$
\hat{\sigma}:=s_{n}\left(r_{1}(\hat{\boldsymbol{\beta}}), \ldots, r_{n}(\hat{\boldsymbol{\beta}})\right)
$$

If we want to compute an intercept as well, we can easily do so by means of

$$
\hat{\alpha}:=t_{n}\left(r_{1}(\hat{\boldsymbol{\beta}}), \ldots, r_{n}(\hat{\boldsymbol{\beta}})\right)
$$

where $t_{n}$ is a univariate location estimator. When using $\hat{\boldsymbol{\beta}}_{\mathrm{LS}}, \hat{\boldsymbol{\beta}}_{\mathrm{LMS}}$, or $\hat{\boldsymbol{\beta}}_{\mathrm{LTS}}$ it is natural to use $t_{\mathrm{LS}},{ }_{\mathrm{LMS}}$, or $t_{\mathrm{LTS}}$ for this. In the case of LTM we can choose any high-breakdown location estimator $t_{n}$ we like, such as the sample median or a more efficient estimator.

The objective function of the LTM belongs to the class of nested L-estimators of scale, which was introduced by Rousseeuw and Croux (1992). Nested L-estimators are defined as

$$
s_{\mathrm{NL}}\left(r_{1}, \ldots, r_{n}\right)=\sum_{k=1}^{n} b_{k}\left\{H\left(r_{i}\right) ; i=1, \ldots, n\right\}_{(k)}
$$

where

$$
H\left(r_{i}\right)=\sum_{k=1}^{n} a_{k}\left\{\left|r_{i}-r_{j}\right| ; j=1, \ldots, n\right\}_{(k)}
$$

and the $a_{k}$ and $b_{k}$ are coefficients. Clearly $s_{\mathrm{NL}}$ is location-free, and well-suited for asymmetric distributions. One can then consider the regression estimators

$$
\hat{\boldsymbol{\beta}}_{N L}=\underset{\boldsymbol{\beta}}{\operatorname{argmin} s_{\mathrm{NL}}}\left(r_{1}(\boldsymbol{\beta}), \ldots, r_{n}(\boldsymbol{\beta})\right) .
$$

Two members of this class are particularly simple. By taking $a_{[n / 2]+1}=1, b_{1}=\ldots=b_{h_{p}}=$ $1 / h_{p}$ and all the other coefficients zero, (1.13) becomes the LTM. A second possibility is 
to take $a_{[n / 2]+1}=1, b_{h_{p}}=1$ and the other coefficients zero, which gives the estimator $S_{n}$ proposed by Rousseeuw and Croux (1993). This yields

$$
\hat{\boldsymbol{\beta}}_{\mathrm{LMM}}=\underset{\boldsymbol{\beta}}{\operatorname{argmin}}\left(\underset{i}{\operatorname{median}}\left\{\underset{j}{\operatorname{median}}\left|r_{i}(\boldsymbol{\beta})-r_{j}(\boldsymbol{\beta})\right| ; 1 \leq i \leq n\right\}\right) .
$$

Here, the outer median stands for the order statistic of rank $h_{p}$ and the inner median for the order statistic of $\operatorname{rank}[n / 2]+1$. We could call (1.14) the least median of medians (LMM) estimator. However, like the LMS, the LMM also has a non-standard convergence. Therefore, we prefer the LTM over the LMM.

In the next section we will prove that the LTM has the same breakdown point as the LMS and the LTS. Moreover, we will see in Section 3 that its maxbias curve is much lower than that of the LTS. In Section 4 we will give an expression for the influence function of estimators of the type (1.13). We see that the LTM has a gaussian efficiency of $22 \%$, whereas the LTS has only 7\%. In Section 5, we show that the computation times of the LTS and LTM are nearly the same, and give simulation results and an example. The simulation and the example also serve as a test vehicle for the comparison with three well-known robust regression estimators. The final section compares the LTM with other recent types of estimators and draws some conclusions.

\section{ROBUSTNESS AT FINITE SAMPLES}

In order to study the finite-sample robustness properties of the estimators (1.13), we first derive a relation between the objective function (1.11) and the objective function of the LMS, given by

$$
{ }^{s} \operatorname{LMS}\left(r_{1}, \ldots, r_{n}\right)=\min _{t}\left\{\left|r_{i}-t\right| ; 1 \leq i \leq n\right\}_{\left(h_{p}\right)} .
$$

This is done in the following lemma (the proofs are given in the appendix):

Lemma 1. If the coefficients $a_{k}$ and $b_{k}$ in (1.11) and (1.12) are such that

1) $a_{k}=0$ and $b_{k}=0$ for all $k>h_{p}$, and

2a) $a_{h_{p}}>0$

or

2b) $b_{h_{p}}>0$ and there exists $[n / 2]+1 \leq k_{0} \leq h_{p}$ for which $a_{k_{0}}>0$; 
then there exist finite constants $\gamma>0$ and $\delta>0$ such that

$$
\gamma s_{L M S}\left(r_{1}, \ldots, r_{n}\right) \leq s_{N L}\left(r_{1}, \ldots, r_{n}\right) \leq \delta s_{L M S}\left(r_{1}, \ldots, r_{n}\right)
$$

for all possible choices of $r_{1}, \ldots, r_{n}$.

Note that the objective functions of the LTM and the LMM satisfy conditions (2.2). We will assume throughout this paper that no $h_{p}$ of the carriers $\mathbf{x}_{i}$ lie on the same affine hyperplane in $I R^{p-1}$. This is equivalent to saying than no $h_{p}$ observations $\left(\mathbf{x}_{i}, y_{i}\right)$ lie on the same vertical affine hyperplane in $I R^{p}$ (that is, a hyperplane containing both $(\mathbf{c}, 0)$ and $(\mathbf{c}, 1)$ for some vector $\mathbf{c}$ ). Then we have that

Theorem 1. If the coefficients of $s_{N L}$ satisfy condition (D.D), then there always exists a solution to

$$
\underset{\boldsymbol{\beta}}{\operatorname{argmin}} s_{N L}\left(r_{i}(\boldsymbol{\beta})\right)
$$

We will also need the following regularity condition on our observations:

Definition: We say that the carriers $\mathbf{x}_{i}$ are in general position if no $p$ of them belong to the same affine hyperplane in $I R^{p-1}$.

The finite-sample breakdown point tells us how many observations may be contaminated before the estimator becomes unbounded (Donoho and Huber 1983). To be precise, the breakdown point of the estimator $T$ at the sample $Z$ is defined as

$$
\varepsilon_{n}^{*}(T, Z)=\min \left\{m / n ; \sup _{Z^{\prime}}\left|T(Z)-T\left(Z^{\prime}\right)\right|=\infty\right\},
$$

where $Z^{\prime}$ is obtained by replacing any $m$ elements of $Z$ by arbitrary values. (Here, $|$.$| is the$ euclidean norm.) We have the following result:

Theorem 2. If the coefficients of $s_{N L}$ satisfy (2.D) and the $\mathrm{x}_{i}$ are in general position, then

$$
\varepsilon_{n}^{*}\left(\hat{\boldsymbol{\beta}}_{N L}, Z\right)=\frac{[(n-p) / 2]+1}{n} .
$$

Note that (2.4) is the maximal possible value for any regression equivariant estimator (Rousseeuw 1984, page 879). Theorem 2 covers the LTM and LMM as special cases. From the general relation between the breakdown point and the exact fit property (Rousseeuw and Leroy 1987, page 123) it immediately follows that: 
Corollary. If the coefficients of $s_{\mathrm{NL}}$ satisfy (2.2), and at least $h_{p}$ observations obey $y_{i}=\boldsymbol{\beta}_{0}^{t} \mathbf{x}_{i}+\alpha_{0}$ exactly and their $\mathbf{x}_{i}$ are in general position, then $\hat{\boldsymbol{\beta}}_{\mathrm{NL}}=\boldsymbol{\beta}_{0}$.

Estimators with a positive breakdown point are particularly useful at the initial (exploratory) stage of analyzing data. Whereas the residuals of zero-breakdown methods like least squares often fail to reveal the presence of multiple outliers, positive-breakdown methods are able to detect substructures of smaller mass than the breakdown point. When we don't know the percentage of inliers in advance, it is safest to start with a $50 \%$ breakdown method. Note that all positive-breakdown estimators (and not only those with $50 \%$ ) share certain properties that are fundamentally different from least squares, and may seem unnatural to a first-time user. For a survey of such features, including the exact fit property mentioned above, see Rousseeuw (1994).

\section{MAXBIAS CURVES}

The breakdown point only gives a single number summary, in that it tells at how much contamination the estimate can become totally wrong. A much richer concept is the maxbias curve. The maxbias curve plots the worst-case bias of the estimator as a function of the percentage $\epsilon$ of contamination, where $\epsilon$ ranges from zero to the breakdown point. This again shows that a good breakdown point is only a necessary condition for robustness, and not a sufficient one (Rousseeuw 1984, page 877). It is possible that a $50 \%$ breakdown estimator has such a large (though bounded) bias under a fraction $\epsilon$ of contamination, that it becomes useless at that $\epsilon$. Examples of such estimators are discussed, in the scale context, by Croux (1994). Therefore, it is essential to compute the maxbias curve.

Since the maxbias curve is an asymptotic concept, we use the functional version of $\hat{\boldsymbol{\beta}}_{N L}$. We need two score-generating functions $h_{1}$ and $h_{2}^{+}$from $[0,1]$ to $[0, \infty[$ such that

$$
b(k)=\int_{(k-1) / n}^{k / n} h_{1}(t) d t \stackrel{n \rightarrow \infty}{\sim} h_{1}(k / n)
$$

and

$$
a(k)=\int_{(k-1) / n}^{k / n} h_{2}^{+}(t) d t \stackrel{n \rightarrow \infty}{\sim} h_{2}^{+}(k / n) .
$$

(Note that $h_{1}$ and $h_{2}^{+}$may contain delta functions, but not at 0 or 1 .) We also denote

$$
\alpha_{1}=\sup \left\{t>0 ; h_{1}(t)>0\right\} \quad \text { and } \quad \alpha_{2}=\sup \left\{t>0 ; h_{2}^{+}(t)>0\right\} \text {. }
$$


Suppose that the observations (x,y) obey a distribution $K$. Denote for every $\boldsymbol{\beta}$ the distribution of $y-\boldsymbol{\beta}^{t} \mathbf{x}$ by $F_{\boldsymbol{\beta}}$, and for every real number $r$ the distribution of $\left|y-\boldsymbol{\beta}^{t} \mathbf{x}-r\right|$ by $F_{\boldsymbol{\beta}, r}^{+}$. Furthermore put $F_{\boldsymbol{\beta}}^{+}:=F_{\boldsymbol{\beta}, 0}^{+}$and $F^{+}:=F_{\mathbf{0}}^{+}$. We define a function $H_{\boldsymbol{\beta}}$ by

$$
H_{\boldsymbol{\beta}}(r)=\int h_{2}^{+}\left(F_{\boldsymbol{\beta}, r}^{+}(t)\right) t d F_{\boldsymbol{\beta}, r}^{+}(t)
$$

which corresponds to (1.12). If $L_{\boldsymbol{\beta}}$ denotes the distribution of $H_{\boldsymbol{\beta}}\left(y-\boldsymbol{\beta}^{t} \mathbf{x}\right)$, then the functional form of the objective function equals

$$
s(\boldsymbol{\beta}, K)=\int h_{1}\left(L_{\boldsymbol{\beta}}(t)\right) t d L_{\boldsymbol{\beta}}(t)
$$

Our regression functional $T(K)$ is then given by

$$
T(K)=\underset{\boldsymbol{\beta}}{\operatorname{argmin}} s(\boldsymbol{\beta}, K) .
$$

For the definition of the maxbias curve we follow the approach of Martin, Yohai and Zamar (1989). Suppose that our model distribution is given by $K_{0}=\left(G_{0}, F_{0}\right)$, where $G_{0}$ is the distribution of the carriers $\mathrm{x}$, and $F_{0}$ of the errors. We may suppose that $T\left(K_{0}\right)=0$ (due to regression equivariance). Consider the neighborhood of $K_{0}$ given by

$$
V_{\varepsilon}=\left\{K ; K=(1-\varepsilon) K_{0}+\varepsilon K^{*}\right\}
$$

where $K^{*}$ ranges over all distributions. Then the maxbias curve at $\epsilon$ is defined as

$$
B_{\varepsilon}(T)=\sup \left\{|T(K)| ; K \in V_{\varepsilon}\right\}
$$

The asymptotic breakdown point may be defined as

$$
\varepsilon^{*}=\inf \left\{\varepsilon ; B_{\varepsilon}(T)=\infty\right\}
$$

We will impose the following conditions on $K_{0}=\left(G_{0}, F_{0}\right)$ :

(G) $G_{0}$ is spherical, $P_{G_{0}}\left(\mathrm{x}^{t} \boldsymbol{\beta}=0\right)=0$ for all $\boldsymbol{\beta} \neq \mathbf{0}$, and for all $\boldsymbol{\beta}$ the distribution of $\boldsymbol{\beta}^{t} \mathrm{x}$ is unimodal;

(F) $F_{0}$ has a symmetric, unimodal and strictly positive density $f_{0}$;

(GF) The function $\boldsymbol{\beta} \rightarrow g_{\boldsymbol{\beta}_{, s}}\left(\left(F_{0, \boldsymbol{\beta}}^{+}\right)^{-1}(t)\right)$ is strictly increasing in $|\boldsymbol{\beta}|$ for $0 \leq s, t \leq 1$, where $g_{\boldsymbol{\beta}, s}(y)$ is defined implicitly by $F_{\boldsymbol{\beta}}\left(y+g_{\boldsymbol{\beta}, s}(y)\right)-F_{\boldsymbol{\beta}}\left(y-g_{\boldsymbol{\beta}, s}(y)\right)=s$. 
Under these conditions, the breakdown point of the functional $T$ in (3.3) equals

$$
\varepsilon^{*}\left(\hat{\boldsymbol{\beta}}_{N L}\right)=\min \left(\max \left(\alpha_{1}, \alpha_{2}\right), \min \left(1-\alpha_{1}, 1-\alpha_{2}\right)\right) .
$$

For the computation of its maxbias curve, we restrict ourselves to the case where $h_{2}^{+}=\delta_{1 / 2}$ and $\alpha_{1} \leq 1 / 2$ in order to simplify the formulas. Note that both LTM and LMM fall within this scope. Then define $g^{+}(r)$ implicitly by

$$
F_{0}\left(r+g^{+}(r)\right)-F_{0}\left(r-g^{+}(r)\right)=\frac{1}{2(1-\varepsilon)},
$$

and $g_{\boldsymbol{\beta}}^{-}(r)$ by

$$
F_{0, \boldsymbol{\beta}}\left(r+g_{\boldsymbol{\beta}}^{-}(r)\right)-F_{0, \boldsymbol{\beta}}\left(r-g_{\boldsymbol{\beta}^{-}}^{-}(r)\right)=\frac{1-2 \varepsilon}{2(1-\varepsilon)} .
$$

Now consider the function

$$
\tilde{h}(\varepsilon,|\boldsymbol{\beta}|)=g_{\boldsymbol{\beta}}^{-}(0) \int_{0}^{\varepsilon} h_{1}(t) d t+I\left(\varepsilon \leq \alpha_{1}\right) \int_{\varepsilon}^{\alpha_{1}} h_{1}(t) g_{\boldsymbol{\beta}^{-}}^{-}\left(\left(F_{0, \boldsymbol{\beta}}^{+}\right)^{-1}\left(\frac{t-\varepsilon}{1-\varepsilon}\right)\right) d t .
$$

(If $\varepsilon>\alpha_{1}$ then the second term vanishes.) Note that, due to condition $(\mathrm{G})$, the right hand side of (3.10) only depends on $\boldsymbol{\beta}$ through $|\boldsymbol{\beta}|$. Furthermore, by Lemma 3 in the Appendix and condition (GF) we see that $\tilde{h}$ is strictly increasing in $|\boldsymbol{\beta}|$. Denote by $\tilde{h}^{-1}$ the inverse of $\tilde{h}$ w.r.t. $|\boldsymbol{\beta}|$, for fixed $\varepsilon$.

Theorem 3. Under conditions $(G),(F)$ and $(G F)$, and with $h_{2}^{+}=\delta_{1 / 2}$ and $\alpha_{1} \leq 1 / 2$, we have

$$
\begin{aligned}
B_{\varepsilon}(T) & =\tilde{h}^{-1}\left(\varepsilon, s_{1}\right) & & \text { for } \varepsilon<1 / 2 \\
& =\infty & & \text { for } \varepsilon \geq 1 / 2
\end{aligned}
$$

where

$$
s_{1}=\int_{0}^{\alpha_{1}} h_{1}(t) g^{+}\left(F_{0}^{-1}\left(\frac{1+t-\varepsilon}{2(1-\varepsilon)}\right)\right) d t .
$$

If $K_{0}$ is the multivariate standard normal distribution (which satisfies conditions $(\mathrm{G})$, $(\mathrm{F})$ and $(\mathrm{GF}))$ it is possible to obtain explicit formulas for $(3.11)$. We will denote $g^{-}=g_{\mathbf{0}}^{-}$. As a first example we will consider the case $h_{1}(t)=I\left(t \leq \alpha_{1}\right)$ and $h_{2}^{+}=\delta_{1 / 2}$. Then we find

$$
B_{\varepsilon}(T)^{2}=\left\{\frac{\int_{0}^{\Phi^{-1}\left(\frac{1+\alpha_{1}-\varepsilon}{2(1-\varepsilon)}\right)} g^{+}(r) d \Phi(r)}{\Phi^{-1}\left(\frac{3-4 \varepsilon}{4(1-\varepsilon)}\right) \frac{\varepsilon}{2(1-\varepsilon)}+\int_{0}^{\Phi^{-1}\left(\frac{1+\alpha_{1}-\varepsilon}{2(1-\varepsilon)}\right)} g^{-}(r) d \Phi(r)}\right\}^{2}-1
$$


for $0 \leq \varepsilon<\frac{1}{2}$. For $\alpha_{1}=1 / 2$ we obtain the maxbias curve of the LTM. As a second example, take $h_{1}(t)=\delta_{\alpha_{1}}$ with $h_{2}^{+}=\delta_{1 / 2}$ and $\alpha_{1} \leq 1 / 2$. Then we obtain the expression

$$
\begin{aligned}
& B_{\varepsilon}(T)^{2}=\left\{\frac{g^{+}\left(\Phi^{-1}\left(\frac{1+\alpha_{1}-\varepsilon}{2(1-\varepsilon)}\right)\right)}{g^{-}\left(\Phi^{-1}\left(\frac{1+\alpha_{1}-2 \varepsilon}{2(1-\varepsilon)}\right)\right)}\right\}^{2}-1 \quad \text { for } 0 \leq \varepsilon \leq \alpha_{1} \\
& =\left\{\frac{g^{+}\left(\Phi^{-1}\left(\frac{1+\alpha_{1}-\varepsilon}{2(1-\varepsilon)}\right)\right.}{\Phi^{-1}\left(\frac{3-4 \varepsilon}{4(1-\varepsilon)}\right)}\right\}^{2}-1 \quad \text { for } 1 / 2>\varepsilon \geq \alpha_{1} \text {. }
\end{aligned}
$$

Taking $\alpha_{1}=1 / 2$ yields the maxbias curve of the LMM. Furthermore, in the limiting case $\alpha_{1} \rightarrow 0$ we recover the maxbias curve of the LMS, which is essentially the lowest possible among all residual admissable estimators (Yohai and Zamar 1993).

Figure 1 shows the maxbias curves of the LMS, LTS, LTM and LMM at the standard normal model distribution $K_{0}=N\left(\mathbf{0}, \mathrm{I}_{p+1}\right)$. We see that the curves have a vertical tangent at 0 , which holds for all regression estimators with dimension-free maxbias curve (He and Simpson 1993). Furthermore, they tend to infinity when $\varepsilon$ tends to 0.5 , the breakdown point of these estimators. We see that the LMS has the lowest maxbias curve. The LTM stays quite close to the LMS, whereas the LTS curve is much higher. The maxbias curve of the LMM lies between that of the LTS and the LTM. The main conclusion is that the LTM estimator is much more robust than the LTS in terms of its maxbias curve (and we will see that its efficiency is better too.)

A referee remarked that nesting doesn't lower the maxbias curve of LMS, whereas it does lower the maxbias curve of LTS. This is because the collection $\left\{H\left(r_{i}\right) ; 1 \leq i \leq n\right\}$ is more homogeneous than the collection $\left\{r_{i}^{2} ; 1 \leq i \leq n\right\}$. That is less important if we take only one order statistic from either collection, yielding LMM and LMS. But the objective function of LTM (obtained by summing many elements of the first collection) does become more stable than that of LTS (obtained by summing many elements of the second collection).

Remark: For Figure 1 we needed an expression for the maxbias curve of the LTS estimator, which to our knowledge has not yet appeared in the literature. For this purpose we computed the maxbias curves of signed R-estimators (Hössjer 1994), which are defined by minimization of the functional

$$
s(\boldsymbol{\beta}, K)=\int h^{+}\left(F_{\boldsymbol{\beta}^{+}}^{(t)}\right)|t|^{k} d F_{\boldsymbol{\beta}}^{+}(t)
$$

where $h^{+}$is a nonnegative score generating function, $F_{\boldsymbol{\beta}}^{+}$is the distribution of $\left|y-\boldsymbol{\beta}^{t} \mathbf{x}\right|$, and 
$k$ is a positive integer. If we define

$$
\tilde{l}(a, b,|\boldsymbol{\beta}|)=\int_{b}^{a+b} h^{+}(t)\left(\left(F_{0, \boldsymbol{\beta}}^{+}\right)^{-1}\left(\frac{t-b}{a}\right)\right)^{k} d t
$$

then we have under conditions $(\mathrm{F})$ and $(\mathrm{G})$ that $\tilde{h}$ depends only on $\boldsymbol{\beta}$ through $|\boldsymbol{\beta}|$ and is strictly increasing in $|\boldsymbol{\beta}|$ by Lemma 3. Denoting by $\tilde{l}^{-1}$ the inverse of $\tilde{l}$ w.r.t. $|\boldsymbol{\beta}|$ gives

$$
\begin{aligned}
B_{\varepsilon}(T) & =\tilde{l}^{-1}(1-\varepsilon, \varepsilon, \tilde{l}(1-\varepsilon, 0,0)) & & \text { for } 0 \leq \varepsilon<\min (\alpha, 1-\alpha) \\
& =\infty & & \text { elsewhere }
\end{aligned}
$$

where $\alpha=\sup \left\{t ; h^{+}(t)>0\right\}$, as shown in the Appendix. In the special case where $h^{+}(t)=$ $I(t \leq 1 / 2)$ and $K_{0}$ is the multivariate standard normal distribution, we obtain

$$
B_{\varepsilon}(T)^{2}=\left\{\frac{\int_{0}^{\Phi^{-1}\left(\frac{3-2 \varepsilon}{4(1-\varepsilon)}\right)}|r|^{k} d \Phi(r)}{\int_{0}^{\Phi^{-1}\left(\frac{3-4 \varepsilon}{4(1-\varepsilon)}\right)}|r|^{k} d \Phi(r)}\right\}^{2}-1
$$

The case $k=2$ corresponds with the maxbias curve of the LTS estimator.

\section{INFLUENCE FUNCTION AND EFFICIENCIES}

Whereas the maxbias curve tells us how much the estimator may react to a given fraction of contamination, the influence function (see Hampel et al 1986) describes the (standardized) effect of a single outlier. Let $K_{0}$ again be our model distribution, and let $K_{\varepsilon}=(1-\varepsilon) K_{0}+$ $\varepsilon \Delta_{\mathbf{x}, y}$ where $\Delta_{\mathbf{x}, y}$ is the distribution which puts all its mass at the point $(\mathbf{x}, y)$. Then the influence function of the functional $T$ is defined as

$$
I F(\mathrm{x}, y)=\lim _{\varepsilon \downarrow 0} \frac{T\left(K_{\varepsilon}\right)-T\left(K_{0}\right)}{\varepsilon} .
$$

Under smoothness assumptions, it is shown in the Appendix that the minimizer $\boldsymbol{\beta}$ of the nested L-scale $s_{\mathrm{NL}}(\boldsymbol{\beta}, K)$ satisfies the necessary condition

$$
\int \tilde{\psi}\left(y-\boldsymbol{\beta}^{t} \mathbf{x}, \boldsymbol{\beta}, K\right) \mathbf{x} d K(\mathbf{x}, y)=\mathbf{0}
$$

(which clearly is not a sufficient condition). Here,

$\tilde{\psi}(r, \boldsymbol{\beta}, K)=E_{K}\left[h_{1}\left(L_{\boldsymbol{\beta}^{(}}\left(H_{\boldsymbol{\beta}}\left(y-\boldsymbol{\beta}^{t} \mathbf{x}\right)\right)\right) \tilde{\mu}\left(y-\boldsymbol{\beta}^{t} \mathbf{x}, r, \boldsymbol{\beta}\right)\right]-E_{K}\left[h_{1}\left(L_{\boldsymbol{\beta}}\left(H_{\boldsymbol{\beta}}(r)\right)\right) \tilde{\mu}\left(r, y-\boldsymbol{\beta}^{t} \mathbf{x}, \boldsymbol{\beta}\right)\right]$ 
and

$$
\tilde{\mu}(r, s, \boldsymbol{\beta})=h_{2}^{+}\left(F_{\boldsymbol{\beta}, r}^{+}(|s-r|)\right) \operatorname{sign}(s-r)
$$

using the notations of the preceding section. We see that $\tilde{\psi}(r, \boldsymbol{\beta}, K)$ depends only on the distribution of $y-\boldsymbol{\beta}^{t} x$. Therefore we can use Lemma 4 of the Appendix, which yields the influence function of the regression functional $T$ that minimizes the nested L-estimator $s_{\mathrm{NL}}$. At the model distribution, where we assume that $T\left(K_{0}\right)=0$ and $E[\mathbf{x}]=0$ (without needing any symmetry this time), the influence function of $T$ becomes

$$
I F(\mathrm{x}, y)=\frac{\tilde{\psi}_{0}(y)}{E_{F_{0}}\left[\tilde{\psi}_{0}^{\prime}(Y)\right]} \Sigma^{-1} \mathbf{x}
$$

with $\boldsymbol{\Sigma}=E\left[\mathbf{x x}^{t}\right]$ and $\tilde{\psi}_{0}(y)=\tilde{\psi}\left(y, \mathbf{0}, K_{0}\right)$. It is possible to rewrite $\tilde{\psi}_{0}(y)$ by means of the transformed score function

$$
\begin{aligned}
h_{2}(t) & =h_{2}^{+}(2 t-1) & & \text { for } 1 / 2 \leq t \leq 1 \\
& =-h_{2}^{+}(1-2 t) & & \text { for } 0 \leq t<1 / 2
\end{aligned}
$$

and the distribution function

$$
G_{y}\left(y_{1}\right)=\left(F_{0}\left(y_{1}\right)-F_{0}\left(2 y-y_{1}\right)+1\right) / 2
$$

which has the property that $y$ is its median. Then we find that

$$
\tilde{\psi}_{0}(y)=E_{F_{0}}\left[h_{1}(L(H(Y))) h_{2}\left(G_{Y}(y)\right)\right]-E_{F_{0}}\left[h_{1}(L(H(y))) h_{2}\left(G_{y}(Y)\right)\right]
$$

where $L=L_{0}$ and $H=H_{0}$. We also obtain another expression for (1.12):

$$
H(y)=\int h_{2}\left(G_{y}\left(y_{1}\right)\right)\left(y_{1}-y\right) d F_{0}(y)
$$

Equations (4.8) and (4.9) are derived in the Appendix.

As a first example, consider the case where both $h_{1}$ and $h_{2}^{+}$are constant and equal to 1 . This corresponds to Wilcoxon scores. Then, with $h_{2}(t)=\operatorname{sign}(t-1 / 2), H(y)=E_{F_{0}}[|y-Y|]$, and $\tilde{\psi}_{0}(y)=2 E_{F_{0}}[\operatorname{sign}(y-Y)]=4\left(F_{0}(y)-1 / 2\right)$, it is easy to see that $(4.5)$ results in

$$
I F(\mathrm{x}, y)=\frac{F_{0}(y)-1 / 2}{\int F_{0}^{\prime}(y)^{2} d y} \Sigma^{-1} \mathrm{x} .
$$

Our main example is the influence function of the LTM, where $h_{1}=I(0 \leq t \leq 1 / 2)$ and $h_{2}=\left(\delta_{3 / 4}-\delta_{1 / 4}\right) / 2$. We will assume here that $F_{0}$ satisfies condition $(\mathrm{F})$ of Section 3 . The Appendix verifies that $H(y)$ is the unique solution of the equation

$$
F_{0}(y+H(y))-F_{0}(y-H(y))=1 / 2 .
$$


Denote $v(z)=z+H(z)$ and its inverse by $v^{-1}$, which is well-defined since $v$ is an increasing function. With the short-hand notations $q=F_{0}^{-1}(3 / 4)$ and $c=H(q)$ we obtain

$$
\begin{aligned}
\tilde{\psi}_{0}(y) & =H^{\prime}(y) I(|y| \leq q)+\frac{I(c-q<y<c+q) f_{0}\left(v^{-1}(y)\right)}{2 f_{0}\left(y-2 v^{-1}(y)\right)} & & \text { for } y \geq 0 \\
& =-\tilde{\psi}_{0}(-y) & & \text { for } y \leq 0
\end{aligned}
$$

Combining (4.10) and (4.11) makes it possible to compute the influence function of the LTM from (4.5). The $I F$ of the LTM is bounded in the response variable $y$, but not in the design variable $\mathrm{x}$. It also contains some discontinuities. This behavior is similar to that of the IF of the LTS. Analogously, the IF of the LMM is similar to that of the LMS, which was discussed in Davies (1993). The IF of the LMM is zero almost everywhere, which is linked to its a non-standard convergence.

We can now compute the asymptotic covariance matrix $V$ from

$$
V=E_{K_{0}}\left[I F(\mathrm{x}, y) I F(\mathrm{x}, y)^{t}\right]
$$

The efficiency of the estimator $\hat{\boldsymbol{\beta}}_{N L}$ at a gaussian error distribution thus becomes

$$
e=\frac{E_{\Phi}\left[\tilde{\psi}_{0}^{\prime}(Y)\right]^{2}}{E_{\Phi}\left[\tilde{\psi}_{0}^{2}(Y)\right]}
$$

By means of numerical integration we find that the gaussian efficiency of the LTM equals $22.8 \%$, which is considerably higher than the $7.1 \%$ gaussian efficiency of the LTS.

\section{COMPUTATION}

For a high-breakdown objective function $s_{n}$ it is quite hard to minimize (1.8). For this we can use the subsampling algorithm (see Rousseeuw and Leroy 1987), which goes as follows. Consider any subset $J$ of $p$ distinct indices from $\{1, \ldots, n\}$. The corresponding trial estimate $\left(\boldsymbol{\beta}_{J}, \alpha_{J}\right)$ is the parameter vector which satisfies $y_{i}=\boldsymbol{\beta}_{J}^{t} \mathbf{x}_{i}+\alpha_{J}$ for all $i$ in $J$. The final estimate is then given by minimizing

$$
s_{n}\left(r_{1}\left(\boldsymbol{\beta}_{J}, \alpha_{J}\right), \ldots, r_{n}\left(\boldsymbol{\beta}_{J}, \alpha_{J}\right)\right)
$$

where $J$ ranges over some or all possible $p$-subsets. We switch from exhaustive search to a random collection $J$ of $p$-subsets when $\left(\begin{array}{l}n \\ p\end{array}\right)$ becomes large (following Table 2 in Rousseeuw and Leroy 1987, page 199). We compute $\hat{\boldsymbol{\beta}}_{n}, \hat{\alpha}_{n}$ and $\hat{\sigma}_{n}$ according to the definitions in Section 1. 
For LMS and LTS this means that the intercept $\alpha_{J}$ is computed at every trial estimate $\boldsymbol{\beta}_{J}$, as described in (Rousseeuw and Leroy 1987, page 201). This type of intercept adjustment was endorsed in the literature (see, e.g., Tichavsky 1991, Hawkins and Simonoff 1993) because of its accuracy. Note that the LTM objective (1.7) is location-free, so $\hat{\alpha}$ LTM only needs to be computed at the end by means of (1.10). Furthermore, we note that in order to obtain consistent scale estimators $\hat{\sigma}$ at gaussian error distributions we have to multiply $s_{n}(\hat{\boldsymbol{\beta}})$ with certain constants (Rousseeuw and Croux 1992). These are 1.4826 (LMS), 2.6477 (LTS), 1.3800 (LTM) and 1.1926 (LMM).

Simulation: In order to study the finite-sample efficiency of the LMS, LTS, LTM and LMM, we performed a simulation study. For certain values of $n$ and $p$ we generated $m=$ 1000 samples $\left\{\left(\mathbf{x}_{i}, y_{i}\right) ; i=1, \ldots, n\right\}$ from a $p$-dimensional standard gaussian distribution, corresponding to a regression model with gaussian errors and both slope and intercept equal to zero. For each sample we computed estimates of the slope, the intercept, and the scale parameter. The finite-sample efficiencies of these estimators are listed in Table 1. (Only one slope coefficient is given, since the others behave similarly). The efficiencies of the scale estimates were obtained by a standardization, as in Rousseeuw and Croux (1993).

We see that the finite-sample efficiencies of the LTM slope are indeed somewhat better than those of the LTS, as expected. But although the LMM has zero asymptotic efficiency, its finite-sample performance is actually better than that of the LTM, at least for the sample sizes considered in Table 1. (The same effect is seen when comparing the LMS with the LTS in the table.) Also note that the efficiency of the intercept and the scale is much better for the LTM and the LMM than for the other estimators. Although the numbers in Table 1 were obtained by the $p$-subset algorithm, we expect the same tendencies to hold for the exact estimates.

We also make a comparison with three other estimators. The first is an S-estimator (Rousseeuw and Yohai 1984) with $50 \%$ breakdown point, using Tukey's biweight $\rho$-function. This estimator was computed with the SURREAL algorithm of Ruppert (1993). The second method starts from a high-breakdown initial estimator, followed by a one-step Generalized M-estimator of the Mallows type (Simpson, Ruppert, and Carroll 1992), which we will call a Mallows one-step (M1S) estimator. We used the LTS as initial regression estimator, and the Minimum Volume Ellipsoid (MVE) method (Rousseeuw 1985) to downweight outliers in the $\mathrm{x}$-space. The generalized M-step used the Huber $\psi$-function with bending constant 
Table 1: Finite-Sample Efficiencies of the LMS, LTS, LTM, and LMM Estimators

\begin{tabular}{|c|c|c|c|c|c|c|c|c|c|c|c|c|c|}
\hline \multirow[b]{2}{*}{$p$} & \multirow[b]{2}{*}{$n$} & \multicolumn{4}{|c|}{$\boldsymbol{\beta}_{p-1}$} & \multicolumn{4}{|c|}{ intercept } & \multicolumn{4}{|c|}{ scale } \\
\hline & & LMS & LTS & LTM & LMM & LMS & LTS & LTM & LMM & LMS & LTS & LTM & LMM \\
\hline \multirow[t]{3}{*}{2} & 20 & 18.7 & 17.7 & 22.0 & 25.2 & 21.6 & 21.7 & 49.7 & 53.0 & 32.5 & 30.5 & 38.2 & 43.9 \\
\hline & 40 & 17.2 & 15.6 & 21.3 & 25.0 & 19.1 & 18.4 & 57.0 & 58.5 & 38.3 & 34.5 & 45.9 & 54.4 \\
\hline & 100 & 15.6 & 13.1 & 21.4 & 23.1 & 14.9 & 13.0 & 58.3 & 59.5 & 40.7 & 33.9 & 48.1 & 56.5 \\
\hline \multirow[t]{3}{*}{3} & 20 & 19.5 & 18.7 & 20.8 & 25.9 & 22.3 & 21.2 & 36.2 & 42.1 & 34.4 & 32.5 & 34.8 & 42.9 \\
\hline & 40 & 19.2 & 17.0 & 22.3 & 25.7 & 19.7 & 19.6 & 48.7 & 50.8 & 38.6 & 35.9 & 43.6 & 51.9 \\
\hline & 100 & 15.7 & 14.0 & 21.1 & 22.4 & 15.0 & 13.7 & 56.2 & 56.1 & 43.2 & 35.6 & 47.8 & 58.1 \\
\hline \multirow[t]{2}{*}{4} & 40 & 16.9 & 16.4 & 19.2 & 22.9 & 19.1 & 17.7 & 41.3 & 44.5 & 35.9 & 31.7 & 38.1 & 46.3 \\
\hline & 100 & 14.1 & 12.7 & 17.2 & 19.9 & 14.3 & 13.4 & 46.2 & 48.6 & 41.1 & 35.5 & 46.0 & 54.1 \\
\hline \multirow[t]{2}{*}{5} & 40 & 18.4 & 16.9 & 18.4 & 20.1 & 19.7 & 19.3 & 33.3 & 36.0 & 39.7 & 34.7 & 39.1 & 47.6 \\
\hline & 100 & 14.2 & 12.3 & 15.6 & 16.0 & 15.8 & 14.2 & 45.7 & 49.5 & 39.6 & 36.3 & 45.9 & 52.0 \\
\hline all & $\infty$ & 00.0 & 07.1 & 22.8 & 00.0 & 00.0 & 07.1 & 63.7 & 63.7 & 36.7 & 30.7 & 52.7 & 58.2 \\
\hline
\end{tabular}

$c=1.345$. The third estimator is similar to M1S, except that we now use a Schweppe type GM-estimator as proposed by Coakley and Hettmansperger (1993). This method will be called a Schweppe one-step (S1S) estimator.

In our experiment we generated 1000 bivariate gaussian data clouds and estimated the slope by each of the considered methods. The results are reported in Table 2. We see that M1S and S1S have the best performance. Note that if we use the new LTM as our highbreakdown initial estimator, the finite-sample efficiencies of these one-step estimators (now denoted as $\mathrm{M}^{*} \mathrm{~S}^{*}$ and $\mathrm{S}_{\mathrm{S}} \mathrm{S}^{*}$ ) are substantially improved.

Computation times: The time needed to compute the objective function $s_{n}$ determines the total computation time, the remainder of the algorithm being the same. The objective functions of the LMS (1.4) and the LTS (1.6) can be computed in $O(n \log n)$ time by means of univariate algorithms (Rousseeuw and Leroy 1987, pages 169-172). For the LTM and the LMM objective functions we use the efficient algorithm of Croux and Rousseeuw (1992), also in $O(n \log n)$ time. Note that although the LMS, LTS, LTM and LMM all have the same computational complexity, this does not necessarily imply that their actual 
Table 2: Comparison of several High-Breakdown Regression Estimators

\begin{tabular}{cccccccccc}
\hline \hline & \multicolumn{1}{c}{ finite-sample efficiency } \\
\cline { 2 - 10 }$n$ & LMS & LTS & LTM & LMM & S & M1S & S1S & M1S $^{*}$ & S1S $^{*}$ \\
\hline 10 & 20.8 & 23.1 & 21.6 & 24.2 & 29.5 & 34.6 & 33.2 & 53.7 & 51.9 \\
20 & 19.9 & 20.0 & 23.2 & 26.2 & 32.0 & 52.9 & 50.8 & 65.8 & 65.5 \\
40 & 16.9 & 14.9 & 21.5 & 23.8 & 26.6 & 67.6 & 68.6 & 74.8 & 73.8 \\
60 & 16.0 & 13.9 & 24.0 & 24.5 & 27.0 & 76.7 & 76.9 & 87.4 & 88.4 \\
80 & 15.3 & 12.8 & 23.3 & 26.0 & 29.3 & 88.0 & 84.9 & 85.5 & 85.2 \\
100 & 13.4 & 13.4 & 20.9 & 22.5 & 30.1 & 81.8 & 85.5 & 85.4 & 86.5 \\
200 & 12.8 & 11.6 & 23.0 & 20.7 & 28.1 & 84.4 & 85.4 & 90.7 & 91.3 \\
\hline
\end{tabular}

computation times are the same in practice, since large constant factors may be involved. Therefore, we calculated the average computation time for the estimation of $\boldsymbol{\beta}, \alpha$ and $\sigma$ over 10 normally generated samples of size $n$, in the model $y_{i}=\boldsymbol{\beta}^{t} x_{i}+\alpha+\sigma e_{i}$ with $p=2$. We applied an exhaustive search over all p-subsets. Figure 2 shows the average computation time (in seconds on a Sun workstation) versus the sample size. We see that the computation of the LMS is the fastest, whereas the LMM, LTS and LTM timings are very close to each other. The main conclusion from this experiment is that the LTM does not need much more computation time than its competitor, the LTS. We expect that the relations between these computation times will be similar even if other search techniques are used to estimate $\boldsymbol{\beta}$ (see, e.g. Bradu and Hawkins 1993, Woodruff and Rocke 1993), because they all include frequent computation of the objective function. The absolute computation times can be substantially smaller when one uses, for example, the feasible subset algorithm of Hawkins (1993). However, for the above comparison between estimators we preferred to use the same algorithm to compute all four of them. An additional advantage of the exhaustive $p$-subset algorithm is that the result is certain to maintain the exact breakdown point (Rousseeuw and Bassett 1991).

Example 1: Robust regression methods are not only used to provide estimates of $\alpha, \boldsymbol{\beta}$, and $\sigma$, but also to detect unexpected structure, which is an important part of data analysis. Let us illustrate this with a data set, provided by E. Trauwaert, from a Belgian factory of nuclear fuel. The data are listed in Table 3. Each row corresponds with a batch of plutonium, and the variables are concentrations of various isotopes: $x_{i 1}$ is the concentration 
of ${ }^{238} \mathrm{PU}, x_{i 2}$ is ${ }^{239} \mathrm{PU}, x_{i 3}$ is ${ }^{240} \mathrm{PU}$, and $y_{i}$ is ${ }^{241} \mathrm{PU}$. Figure 3 a plots the standardized residuals $r_{i} / \hat{\sigma}$ versus the robust distances $\mathrm{RD}\left(\mathbf{x}_{i}\right)$. The residuals were obtained by the LTM regression method, using an exhaustive search over all $p$-subsets. The distances $\mathrm{RD}\left(\mathrm{x}_{i}\right)$ are based on the MVE estimator, as described in Rousseeuw and Leroy (1987, page 266-269). They measure in a robust way how far $\mathbf{x}_{i}=\left(x_{i 1}, x_{i 2}, x_{i 3}\right)$ is from the 'center' of the $\mathrm{x}$-cloud. Plotting robust residuals versus $\mathrm{RD}\left(\mathbf{x}_{i}\right)$ was proposed in (Rousseeuw and van Zomeren 1990).

In Figure 3a, many points have small residuals and small $\operatorname{RD}\left(\mathbf{x}_{i}\right)$. Moreover, three points have small $\mathrm{RD}\left(\mathbf{x}_{i}\right)$ but large $\left|r_{i} / \hat{\sigma}\right|$, hence they are vertical outliers. The points with large $\operatorname{RD}\left(\mathbf{x}_{i}\right)$ we call leverage points, and two types can be distinguished. Those for which $\left|r_{i} / \hat{\sigma}\right|$ is large at the same time we call bad leverage points ("bad" because they have an adverse effect on least squares). In contrast, those with small $\left|r_{i} / \hat{\sigma}\right|$ we call good leverage points ("good" because they can improve the precision of the regression coefficients). Note that a plot of LS residuals versus classical Mahalanobis distances $\operatorname{MD}\left(x_{i}\right)$ reveals no structure here (Figure 3b). In view of the unusual pattern in Figure 3a, we may conclude that the data set is not homogeneous. For instance, we could partition the data into a group of nine observations (9-16 and 21) with large negative residuals, a second group of six observations (22 and 29-33) with large positive residuals, and the remaining group with residuals inside the tolerance band. Note that each of these groups is "linear" in the sense that its $\left(\mathbf{x}_{i}, y_{i}\right)$ belong to a thickened hyperplane, which is different from the hyperellipsoidal type of groups that most clustering methods aim for.

A referee asked to add plots of the LTM residuals versus each of the explanatory variables (Figure 3c). In the case of LS residuals, plots of this type are often used to verify whether the model is well specified. Recently it has been debated whether robust residual plots can be used for the same purpose (Cook, Hawkins and Weisberg 1992; McKean, Sheather and Hettmansperger 1993; Davies 1994). To settle this matter would require a theory for the behavior of robust residual plots under various types of model violations. To be fair, it should be noted that the existing theory for using LS residual plots for model specification is not complete either, since it is essentially restricted to situations with little or no contamination.

A different question is to the relative advantages of figures like $3 \mathrm{a}$ and $3 \mathrm{c}$ for purposes of outlier detection. Both figures plot the same robust residuals on the vertical axis, only the horizontal axes differ. Theoretically, Figure 3c contains the most information: when the points are labelled we can actually compute the $\operatorname{RD}\left(x_{i}\right)$ from it, yielding Figure $3 \mathrm{a}$. On the 
Table 3: Plutonium Data Set, with Robust Distances $\mathrm{RD}\left(\mathrm{x}_{i}\right)$ obtained by applying the MVE in $\mathbf{x}$-space, and with Standardized Residuals $r_{i} / \hat{\sigma}$ obtained by LTM Regression

\begin{tabular}{|c|c|c|c|c|c|c|}
\hline$i$ & $x_{i 1}$ & $x_{i 2}$ & $x_{i 3}$ & $y_{i}$ & $\mathrm{RD}\left(\mathrm{x}_{i}\right)$ & $r_{i} / \hat{\sigma}$ \\
\hline 1 & 0.126 & 75.804 & 21.204 & 2.180 & 1.00 & 0.00 \\
\hline 2 & 0.133 & 75.515 & 21.408 & 2.240 & 1.11 & 0.10 \\
\hline 3 & 0.127 & 75.175 & 21.668 & 2.305 & 1.04 & 0.00 \\
\hline 4 & 0.156 & 78.872 & 18.428 & 1.906 & 1.26 & 0.02 \\
\hline 5 & 0.503 & 73.317 & 20.223 & 4.128 & 0.92 & -0.55 \\
\hline 6 & 0.113 & 79.116 & 18.548 & 1.690 & 1.08 & -0.17 \\
\hline 7 & 0.129 & 75.751 & 21.162 & 2.260 & 0.80 & 0.17 \\
\hline 8 & 0.124 & 75.326 & 21.557 & 2.282 & 0.99 & 0.03 \\
\hline 9 & 1.022 & 63.287 & 24.493 & 6.990 & 1.02 & -9.54 \\
\hline 10 & 1.412 & 59.553 & 25.576 & 8.027 & 6.84 & -13.78 \\
\hline 11 & 1.533 & 58.688 & 25.719 & 8.279 & 9.04 & -14.98 \\
\hline 12 & 1.534 & 58.758 & 25.692 & 8.261 & 9.26 & -14.74 \\
\hline 13 & 1.437 & 59.728 & 25.146 & 8.377 & 6.44 & -10.91 \\
\hline 14 & 1.439 & 59.544 & 25.126 & 8.569 & 5.58 & -10.36 \\
\hline 15 & 1.375 & 59.877 & 25.128 & 8.428 & 4.28 & -10.35 \\
\hline 16 & 1.153 & 61.182 & 25.100 & 7.802 & 1.00 & -11.02 \\
\hline 17 & 0.201 & 78.244 & 18.488 & 2.351 & 1.00 & 1.31 \\
\hline 18 & 0.176 & 78.166 & 18.629 & 2.365 & 0.92 & 1.56 \\
\hline 19 & 0.239 & 74.254 & 21.515 & 2.901 & 0.63 & -0.39 \\
\hline 20 & 0.102 & 79.840 & 17.872 & 1.674 & 1.06 & -0.17 \\
\hline 21 & 1.070 & 62.455 & 24.656 & 7.512 & 0.91 & -8.18 \\
\hline 22 & 0.851 & 73.189 & 18.285 & 5.597 & 5.50 & 6.77 \\
\hline 23 & 0.125 & 75.968 & 20.794 & 2.407 & 1.13 & 0.50 \\
\hline 24 & 0.142 & 75.957 & 20.867 & 2.341 & 0.62 & 0.69 \\
\hline 25 & 0.352 & 72.885 & 21.718 & 3.618 & 0.38 & -0.03 \\
\hline 26 & 0.351 & 72.907 & 21.721 & 3.601 & 0.39 & -0.02 \\
\hline 27 & 0.346 & 72.919 & 21.713 & 3.600 & 0.43 & -0.12 \\
\hline 28 & 0.217 & 76.089 & 20.225 & 2.556 & 0.65 & 0.08 \\
\hline 29 & 1.068 & 70.129 & 18.573 & 7.689 & 3.69 & 11.86 \\
\hline 30 & 1.171 & 69.273 & 18.633 & 8.300 & 4.45 & 14.54 \\
\hline 31 & 1.213 & 69.147 & 18.640 & 8.363 & 5.46 & 15.23 \\
\hline 32 & 1.226 & 68.294 & 18.869 & 8.826 & 4.03 & 15.32 \\
\hline 33 & 1.111 & 71.076 & 18.122 & 7.248 & 6.70 & 12.13 \\
\hline 34 & 0.183 & 75.714 & 20.750 & 2.488 & 0.49 & -0.11 \\
\hline 35 & 0.162 & 76.150 & 20.345 & 2.524 & 1.00 & 0.19 \\
\hline 36 & 0.113 & 77.845 & 19.108 & 2.275 & 2.14 & 0.37 \\
\hline 37 & 1.309 & 62.382 & 22.754 & 9.311 & 2.01 & 2.06 \\
\hline 38 & 1.638 & 60.112 & 23.320 & 9.972 & 7.44 & 1.31 \\
\hline 39 & 1.589 & 60.519 & 23.128 & 9.970 & 6.16 & 2.38 \\
\hline 40 & 1.411 & 61.585 & 23.133 & 9.339 & 3.33 & 0.50 \\
\hline 41 & 1.457 & 61.332 & 23.239 & 9.321 & 4.61 & -0.17 \\
\hline 42 & 0.397 & 72.291 & 21.761 & 3.836 & 0.62 & -1.82 \\
\hline 43 & 0.328 & 73.451 & 21.429 & 3.419 & 0.31 & -0.46 \\
\hline 44 & 0.242 & 74.888 & 20.939 & 2.875 & 0.34 & -0.05 \\
\hline 45 & 1.367 & 60.507 & 23.603 & 9.839 & 2.58 & -0.17 \\
\hline
\end{tabular}


Table 4: Estimates for the plutonium data

\begin{tabular}{cccccc}
\hline \hline estimator & $\hat{\alpha}$ & $\hat{\boldsymbol{\beta}}_{1}$ & $\hat{\boldsymbol{\beta}}_{2}$ & $\hat{\boldsymbol{\beta}}_{3}$ & $100 \times \hat{\boldsymbol{\sigma}}$ \\
\hline LMS & 71.5 & -1.30 & -0.71 & -0.71 & 6.20 \\
LTS & 77.1 & -1.82 & -0.77 & -0.77 & 6.93 \\
LTM & 74.3 & -1.55 & -0.74 & -0.74 & 6.27 \\
LMM & 71.5 & -1.33 & -0.72 & -0.70 & 9.02 \\
S & 73.6 & -1.45 & -0.73 & -0.74 & 9.92 \\
M1S & 78.4 & -1.67 & -0.77 & -0.83 & 6.85 \\
S1S & 80.3 & -1.85 & -0.78 & -0.86 & 6.85 \\
\hline
\end{tabular}

other hand, we recommend drawing Figure $3 \mathrm{a}$ as well, because it combines much information (including the fourfold classification into regular observations, vertical outliers, good and bad leverage points) in a single plot, which is useful to a human viewer. In particular, it may highlight outliers in $\mathrm{x}$-space which are not visible with the individual regressors.

Table 4 gives an overview of the estimates produced by the previously discussed methods. We see that the more efficient methods yield estimates close to the S1S results. All the estimators identify cases 9-16, 21-22, 29-33 as clear outliers, whereas the observations 20, 37 and 39 were sometimes at the border line, with standardized residuals between 2.5 and 3 .

Example 2: Let us now apply the LTM to data with an asymmetric error distribution. Figure 4 contains 30 points which where generated according to $y_{i}=x_{i}+2+\varepsilon_{i}$ where the $\mathbf{x}_{i}$ were generated from a gaussian distribution with mean 4 and unit standard deviation, and where $-2 \varepsilon_{i}$ has a standard exponential distribution. To these points we added 10 severe outliers. We estimate the slope by $\hat{\boldsymbol{\beta}}_{\text {LTM }}$ given in (1.7), and the scale parameter by $\hat{\sigma}=c s_{n}\left(\hat{\boldsymbol{\beta}}_{\mathrm{LTM}}\right)$, where $c=2.189$ is the consistency factor for exponential distributions. Instead of (1.10) we now use

$$
\hat{\alpha}=\underset{i}{\operatorname{median}}\left(y_{i}-\hat{\boldsymbol{\beta}}_{\mathrm{LTM}} x_{i}\right)+\hat{\sigma} \ln 2
$$

which is robust and consistent for the location parameter of this error distribution. When using the least squares slope, we estimated $\alpha$ by the maximum likelihood estimator (MLE):

$$
\hat{\alpha}_{\mathrm{ML}}=\max _{i}\left(y_{i}-\hat{\boldsymbol{\beta}} x_{i}\right)
$$

which is very efficient at the exponential model, but not robust. Figure 5 contains both residual plots. We see that the LTM provides a good fit to the majority and finds the 
Table 5: Average slope estimate over 100 asymmetric data sets

\begin{tabular}{ccccccccc}
\hline \hline slope estimator & LMS & LTS & LTM & LMM & S & M1S & S1S & MLE \\
\hline average & 0.981 & 0.950 & 0.969 & 0.987 & 0.993 & 0.240 & 0.246 & -0.305 \\
standard error & 0.022 & 0.031 & 0.028 & 0.023 & 0.017 & 0.028 & 0.030 & 0.008 \\
\hline
\end{tabular}

outliers, which is not the case for LS. The LTM estimates are $\hat{\alpha}=2.68$ and $\hat{\boldsymbol{\beta}}=0.85$, whereas the LS approach with MLE intercept yields $\hat{\alpha}=10.30$ and $\hat{\boldsymbol{\beta}}=-0.37$. If we apply the second method only to the 30 good observations we obtain $\hat{\alpha}=2.17$ and $\hat{\boldsymbol{\beta}}=0.96$, which is quite close to the LTM estimates.

This example provides another test vehicle for comparing the estimators under consideration. We simulated 100 configurations according to the same model. For each method, Table 5 shows the average of the slope estimates over the 100 samples. (We limit ourselves to the slope, since the intercept and the scale can always be estimated afterwards, whatever method was used for the slope.) We see that all the positive-breakdown estimators in the study yield similar results, except for M1S and S1S. The latter are biased because the leverage points receive an $\mathrm{x}$-based weight of $v_{i}=\min \left(1, \chi_{p, 0.95}^{2} / \mathrm{RD}^{2}\left(\mathrm{x}_{i}\right)\right) \approx 0.5$ which implies that they still have a large effect in the GM step. One way to avoid this would be to downweight leverage points more strongly, e.g. by putting $v_{i}=I\left(\mathrm{RD}^{2}\left(\mathrm{x}_{i}\right) \leq \chi_{p, 0.95}^{2}\right)$. A second possibility is to replace the monotone $\psi$-function in the GM-step by a redescending $\psi$. This is similar to carrying out a reweighted least squares (RLS) step using a redescending weight like $w_{i}=I\left(\left|r_{i} / \hat{\sigma}\right| \leq 2.5\right)$ based on the initial residuals, as implemented in PROGRESS (Rousseeuw 1984; Rousseeuw and Leroy 1987).

\section{DISCUSSION}

Instead of minimizing a nested L-statistic, one could also minimize a nested $M$-estimator of scale (Rousseeuw and Croux, 1992). The latter are defined by

$$
\frac{1}{n} \sum_{i=1}^{n} \rho_{1}\left(\frac{H\left(r_{i}\right)}{s_{n}}\right)=k_{1}
$$

where $H\left(r_{i}\right)$ depends on the data through

$$
\frac{1}{n} \sum_{j=1}^{n} \rho_{2}\left(\frac{r_{i}-r_{j}}{H\left(r_{i}\right)}\right)=k_{2} .
$$


As in the case of S-estimators (Rousseeuw and Yohai 1984), the $\rho_{i}$ are even functions, increasing on the positive numbers, with $\rho_{i}(0)=0$ and $E_{F_{0}}\left[\rho_{i}\right]=k_{i}(i=1,2)$. The regression slope estimators $\hat{\boldsymbol{\beta}}_{\mathrm{NS}}$ obtained by minimizing (6.1) might therefore be called nested $S$ estimators. For their influence function we use the same notation as in Section 4 and put $\psi_{i}=\rho_{i}^{\prime}(i=1,2)$, yielding

$$
I F(\mathrm{x}, y)=\frac{\psi(y)}{E_{F_{0}}\left[\psi^{\prime}(Y)\right]} \Sigma^{-1} \mathbf{x}
$$

where

$$
\psi(y)=E_{F_{0}}\left[\mu(y) \psi_{2}\left(\frac{y-Y}{H(y)}\right)\right]+E_{F_{0}}\left[\mu(Y) \psi_{2}\left(\frac{y-Y}{H(Y)}\right)\right]
$$

and

$$
\mu(y)=\frac{\psi_{1}\left(H(y) / s\left(F_{0}\right)\right)}{E_{F_{0}}\left[\psi_{2}\left(\frac{y-Y}{H(y)}\right)\left(\frac{y-Y}{H(y)}\right)\right]} .
$$

Choosing $\rho_{1}(r)=\rho_{2}(r)=|r|$ corresponds to Wilcoxon scores. On the other hand, by taking $\rho_{1}(r)=\min (|r|, 1)$ and $\rho_{2}(r)=I(|r|>1)$ we obtain an estimator with the same influence function as the LTM (after tedious calculations).

For another example of a nested S-estimator take $\rho_{2}(t)=I(|t| \geq 1 / 2)$, hence $H\left(r_{i}\right)=$ $\operatorname{median}_{j}\left|r_{i}-r_{j}\right|$. By choosing $\rho_{1}$ appropriately, (6.1) becomes the $50 \%$ breakdown biweight M-estimator on these $H\left(r_{i}\right)$. This makes it possible to apply some features of the algorithm of Ruppert (1993) for S-estimators. In this way we obtain a $50 \%$ breakdown regression estimator with computation time comparable to the LTM, but with about $10 \%$ higher efficiency. We didn't investigate the class of nested S-estimators in more detail because of their complex definition, since the goal of this article was to produce a simple initial estimator. For the same reason we don't consider S-estimators as competitors of the LTM method.

One might argue that the LTM should not be compared to the LTS, which contains squares, but rather with the least trimmed absolute deviation estimator (LTAD) defined as

$$
\hat{\boldsymbol{\beta}}_{\mathrm{LTAD}}=\underset{\boldsymbol{\beta}}{\operatorname{argmin}} \frac{1}{h_{p}} \sum_{i=1}^{h_{p}}\left|r_{i}(\boldsymbol{\beta})-t_{\mathrm{LTAD}}\left(r_{1}(\boldsymbol{\beta}), \ldots, r_{n}(\boldsymbol{\beta})\right)\right|
$$

where

$$
t_{\operatorname{LTAD}}\left(r_{1}, \ldots, r_{n}\right)=\underset{\alpha}{\operatorname{argmin}} \frac{1}{h_{p}} \sum_{k=1}^{h_{p}}\left\{\left|r_{i}-\alpha\right| ; i=1, \ldots, n\right\}_{(k)} .
$$

The influence function of the univariate location estimator (6.7) was obtained by Tableman (1994). Like the LTS, the LTAD regression estimator is aimed at symmetric error distributions because its objective is location-based. From Hössjer (1994) it follows that the LTAD 
is asymptotically normal with a gaussian efficiency of $5.3 \%$, which is less than that of the LTS. The maxbias curve of the LTAD is given by formula (3.17) above, with $k=1$. It turns out that the LTAD has a smaller bias than the LTS, but still its maxbias curve is much higher than that of the LTM.

Another highly robust regression method with a simple and explicit objective function is the least quartile difference (LQD) estimator (Croux, Rousseeuw and Hössjer 1994), defined as

$$
\hat{\boldsymbol{\beta}}_{\mathrm{LQD}}=\underset{\boldsymbol{\beta}}{\operatorname{argmin}}\left\{\left|r_{i}-r_{j}\right| ; i<j\right\}_{\left(\left(\begin{array}{c}
h_{p} \\
2
\end{array}\right)\right)} \cdot
$$

The LQD has a $50 \%$ breakdown point, and a somewhat higher maxbias curve than the LTM. Its gaussian efficiency is high (67.1\%), although it looses a lot of efficiency at finite samples. However, the computation time for the LQD is twice as large as for the LTM. A more detailed comparison between the LQD and the LTM can be found in (Croux and Rousseeuw 1994). The LQD belongs to the class of Generalized S-estimators (Croux, Rousseeuw and Hössjer 1994), which are different from nested S-estimators. Generalized S-estimators are more complicated and harder to compute than S-estimators, but they are able to attain a higher efficiency.

Making a choice between the available high-breakdown estimators is not easy, because some conflicting aims are involved. We think that the methods proposed in this paper, defined by minimizing nested L-statistics, form a new class whose properties deserve to be investigated. Their location-free objective functions are appealing, because they allow to fit models with asymmetric error distributions. One member of this new class, the LTM, combines a simple explicit objective function, a $50 \%$ breakdown point, and a very low maxbias curve. Its gaussian efficiency of $22 \%$ compares favorably with the LTS, whereas it does not take much longer to compute. Therefore, we think that the LTM can be very useful, e.g. as an initial estimator for two-stage procedures such as those of Simpson, Ruppert and Carroll (1992) and Coakley and Hettmansperger (1993), as illustrated in Table 2.

\section{Appendix A Proofs and Computations of Section 2}

Proof of Lemma 1. It is clear from (1.4) that s LMS equals half of the length of the 
shortest halfsample, that is

$$
{ }^{s} \operatorname{LMS}=\frac{1}{2} \min \left\{\left|r_{\left(i+h_{p}-1\right)}-r_{(i)}\right| ; 1 \leq i \leq n-h_{p}+1\right\} .
$$

Consider an observation $r_{i}$ which belongs to $\tilde{I}=\left[t_{\mathrm{LMS}}-s_{\mathrm{LMS}},{ }_{\mathrm{LMS}}+{ }^{s} \mathrm{LMS}\right]$. Then we know that for every $r_{j}$ in $\tilde{I}$ it holds that $\left|r_{i}-r_{j}\right| \leq 2 s$ LMS. Since there are at least $h_{p}$ observations $r_{j}$ in $\tilde{I}$, and $a_{k}=0$ for $k>h_{p}$ we have that

$$
H\left(r_{i}\right)=\sum_{k=1}^{h_{p}} a_{k}\left\{\left|r_{i}-r_{j}\right| ; 1 \leq j \leq n\right\}_{(k)} \leq 2\left(\sum_{k=1}^{h_{p}} a_{k}\right){ }^{s} \mathrm{LMS} \cdot
$$

The inequality (A.1) holds for the $h_{p}$ observations $r_{i}$ in $\tilde{I}$. Together with the condition $b_{k}=0$ for $k>h_{p}$, it then follows from (A.1) that

$$
s_{\mathrm{NL}}=\sum_{k=1}^{h_{p}} b_{k}\left\{H\left(r_{i}\right) ; i=1, \ldots, n\right\}_{(k)} \leq 2\left(\sum_{k=1}^{h_{p}} b_{k}\right)\left(\sum_{k=1}^{h_{p}} a_{k}\right) s_{\mathrm{LMS}} \cdot
$$

From the above inequality it follows that there is a positive $\delta$ such that $s_{\mathrm{NL}} \leq \delta s_{\mathrm{LMS}}$.

To prove the other inequality, suppose first that condition (2a) is fulfilled. Defining $\tilde{I}_{i}=\left[r_{i}-\left\{\left|r_{i}-r_{j}\right| ; 1 \leq j \leq n\right\}_{\left(h_{p}\right)}, r_{i}+\left\{\left|r_{i}-r_{j}\right| ; 1 \leq j \leq n\right\}_{\left(h_{p}\right)}\right]$, we know that the length of $\tilde{I}_{i}$ is at least $2 s_{\mathrm{LMS}}$, since $\tilde{I}_{i}$ contains at least $h_{p}$ observations. From this it follows that

$$
H\left(r_{i}\right) \geq a_{h_{p}}\left\{\left|r_{i}-r_{j}\right| ; 1 \leq j \leq n\right\}_{\left(h_{p}\right)} \geq a_{h_{p}} s \mathrm{LMS}
$$

for each $i$. Taking $\gamma=\left(\sum_{k=1}^{h_{p}} b_{k}\right) a_{h_{p}}$ gives the desired result.

Now assume that condition (2b) is fulfilled. If $k \geq[n / 2]+1$ then the interval $\left[r_{i}-\left\{\mid r_{i}-\right.\right.$ $\left.\left.r_{j} \mid ; 1 \leq j \leq n\right\}_{(k)}, r_{i}+\left\{\left|r_{i}-r_{j}\right| ; 1 \leq j \leq n\right\}_{(k)}\right]$ has to contain the sample median. With $k_{0} \geq[n / 2]+1$ such that $a_{k_{0}}>0$, it follows that

$$
H\left(r_{i}\right)=\sum_{k=1}^{h_{p}} a_{k}\left\{\left|r_{i}-r_{j}\right| ; 1 \leq j \leq n\right\}_{(k)} \geq a_{k_{0}}\left\{\left|r_{i}-r_{j}\right| ; 1 \leq j \leq n\right\}_{\left(k_{0}\right)} \geq a_{k_{0}}\left|r_{i}-\underset{j}{\operatorname{median}} r_{j}\right|
$$

for every $i$. From this it follows that

$$
\begin{aligned}
& s_{\mathrm{NL}}=\sum_{k=1}^{h_{p}} b_{k}\left\{H\left(r_{i}\right) ; i=1, \ldots, n\right\}_{(k)} \\
& \geq b_{h_{p}}\left\{H\left(r_{i}\right) ; i=1, \ldots, n\right\}_{\left(h_{p}\right)} \\
& \geq b_{h_{p}} a_{k_{0}}\left\{\left|r_{i}-\underset{j}{\operatorname{median}} r_{j}\right| ; 1 \leq i \leq n\right\}_{\left(h_{p}\right)} \\
& \geq b_{h_{p}} a_{k_{0}}\left\{\left|r_{i}-t_{\mathrm{LMS}}\right| ; 1 \leq i \leq n\right\}_{\left(h_{p}\right)} \\
& =b_{h_{p}} a_{k_{0}} s \mathrm{LMS} \text {. }
\end{aligned}
$$


Choosing $\gamma=b_{h_{p}} a_{k_{0}}$ completes the proof.

Proof of Theorem 1. Let $\gamma$ and $\delta$ be the positive constants given by Lemma 1 . Denote $M=\max _{i}\left|y_{i}\right|$ and $\tilde{T}_{Y}=\tilde{T}\left(y_{1}, \ldots, y_{n}\right)$. Then it is immediate to see that

$$
s_{n}(\mathbf{0}) \leq \delta\left(M+\tilde{T}_{Y}\right)
$$

Since no $h_{p}$ observations lie on the same vertical hyperplane, we have

$$
\inf _{\alpha,|\boldsymbol{\beta}|=1}\left\{\left|\boldsymbol{\beta}^{t} u_{i}+\alpha\right| ; 1 \leq i \leq n\right\}_{\left(h_{p}\right)}=m>0
$$

Taking $|\boldsymbol{\beta}|>c=\left(M+\frac{\delta}{\gamma}\left(\tilde{T}_{Y}+M\right)\right) / m$ yields

$$
\left|r_{i}(\boldsymbol{\beta}, \alpha)\right|=\left|y_{i}-\boldsymbol{\beta}^{t} \mathbf{x}_{i}-\tilde{T}_{Y}\right| \geq|| y_{i}|-| \boldsymbol{\beta}^{t} \mathbf{x}_{i}+\tilde{T}_{Y}|| \geq|\boldsymbol{\beta}| m-M>0
$$

at least $n-h_{p}+1$ times. Therefore $\left\{\left|r_{i}-\tilde{T}_{Y}\right| ; 1 \leq i \leq n\right\}_{\left(h_{p}\right)} \geq|\boldsymbol{\beta}| m-M$, from which it follows that

$$
s_{n}(\boldsymbol{\beta}) \geq \gamma(|\boldsymbol{\beta}| m-M) \geq s_{n}(\mathbf{0})
$$

The above inequality implies that $\boldsymbol{\beta} \rightarrow s_{n}(\boldsymbol{\beta})$ has an infimum inside the compact ball with radius $c$. Since the objective function $s_{\mathrm{NL}}$ is based on continuous functions of order statistics, it is continuous in $r_{1}, \ldots, r_{n}$. Therefore $\boldsymbol{\beta} \rightarrow s_{n}(\boldsymbol{\beta})$ is continuous and attains a minimum (not necessarily unique) inside this ball.

Proof of Theorem 2. Application of the following lemma with $\tilde{T}=t_{\mathrm{LMS}}$, combined with Lemma 1, proves the theorem.

Lemma 2. Suppose that there exists a location estimator $\tilde{T}$ such that $\varepsilon_{n}^{*}(\tilde{T}) \geq([(n-p) / 2]+$ $1) / n$. Suppose further that there exist constants $\gamma>0$ and $\delta>0$ such that

$\gamma\left\{\left|r_{i}-\tilde{T}\left(r_{1}, \ldots, r_{n}\right)\right| ; 1 \leq i \leq n\right\}_{\left(h_{p}\right)} \leq s_{n}\left(r_{1}, \ldots, r_{n}\right) \leq \delta\left\{\left|r_{i}-\tilde{T}\left(r_{1}, \ldots, r_{n}\right)\right| ; 1 \leq i \leq n\right\}_{\left(h_{p}\right)}$

for any set of numbers $r_{1}, \ldots, r_{n}$. Then, if the $\mathrm{x}_{i}$ are in general position,

$$
\varepsilon_{n}^{*}(\hat{\boldsymbol{\beta}}, Z)=\frac{[(n-p) / 2]+1}{n}
$$

for the regression estimator $\hat{\boldsymbol{\beta}}$ with objective function $s_{n}$. 
Proof. It suffices to prove that $\varepsilon_{n}^{*}(\hat{\boldsymbol{\beta}}, Z) \geq([(n-p) / 2]+1) / n$, since $[(n-p) / 2]+1$ is the maximal value for the breakdown point of a regression equivariant estimator. Suppose w.l.o.g. (due to regression equivariance) that $\hat{\boldsymbol{\beta}}$ based on the original sample $Z$ equals zero. Denote $M_{Y}=\max _{i}\left|y_{i}\right|$. Now construct a sample $Z^{\prime}$ by replacing $[(n-p) / 2]$ observations from $Z$. The lower bound on the breakdown point of $\tilde{T}$ implies that there exists an $\tilde{M}$, only depending on Z, such that $\left|\tilde{T}\left(y_{1}^{\prime}, \ldots, y_{n}^{\prime}\right)\right| \leq \tilde{M}$. Denote $M^{*}=M_{Y}+\tilde{M}$ and put $\rho=\frac{1}{2} \inf \left\{\tau>0\right.$; there exists a $(p-2)$ dimensional affine hyperplane $V$ in $I R^{p-1}$ such that $V^{\tau}$ contains at least $p$ observations $\left.\mathbf{x}_{i}\right\}$,

where $V^{\tau}$ is the collection of all points with distance less than $\tau$ from $V$. Since the observations are in general position, $\rho>0$. Now take any $\boldsymbol{\beta}$ with

$$
|\boldsymbol{\beta}| \geq\left(\frac{\delta}{\gamma} M^{*}+M_{Y}\right) / \rho
$$

where $\gamma$ and $\delta$ are given by (A.2). Denote $r_{i}^{\prime}(\boldsymbol{\beta})=y_{i}^{\prime}-\boldsymbol{\beta}^{t} \mathbf{x}_{i}^{\prime}$ yields

$$
\left|r_{i}^{\prime}(\mathbf{0})-\tilde{T}\left(r_{1}^{\prime}(\mathbf{0}), \ldots, r_{n}^{\prime}(\mathbf{0})\right)\right|=\left|y_{i}-\tilde{T}\left(y_{1}^{\prime}, \ldots, y_{n}^{\prime}\right)\right| \leq M_{Y}+\tilde{M}=M^{*}
$$

at least $n-[(n-p) / 2]=h_{p}$ times. Using $($ A.2 $)$ then gives that

$$
s_{n}\left(r_{1}^{\prime}(\mathbf{0}), \ldots, r_{n}^{\prime}(\mathbf{0})\right) \leq \delta M^{*}
$$

Denote $H: y=\boldsymbol{\beta}^{t} \mathbf{x}+\alpha^{\prime}$ where $\alpha^{\prime}=\tilde{T}\left(r_{1}^{\prime}(\boldsymbol{\beta}), \ldots, r_{n}^{\prime}(\boldsymbol{\beta})\right)$. The number of "good points" not belonging to $L=(H \cap(y=0))^{\rho}$ is at least $n-[(n-p) / 2]-(p-1)=h_{p}-p+1$, due to the definition of $\rho$. For those points it holds that

$$
\begin{aligned}
\left|r_{i}^{\prime}(\boldsymbol{\beta})-\tilde{T}\left(r_{1}^{\prime}(\boldsymbol{\beta}), \ldots, r_{n}^{\prime}(\boldsymbol{\beta})\right)\right| & =\left|y_{i}-\boldsymbol{\beta}^{t} \mathbf{x}-\alpha^{\prime}\right| \\
& \geq|| y_{i}|-| \boldsymbol{\beta}^{t} \mathbf{x}_{i}+\alpha^{\prime} \| \\
& >\rho|\boldsymbol{\beta}|-M_{Y}
\end{aligned}
$$

since $\left|y_{i}\right| \leq M_{Y}$ and $\left|\boldsymbol{\beta}^{t} \mathbf{x}+\alpha^{\prime}\right| \geq \rho|\boldsymbol{\beta}|$. (This last inequality can be seen as follows. Take the point $\mathbf{x}$ on $L$ closest to $\mathbf{x}_{i}$. Then $\left|\boldsymbol{\beta}^{t} \mathbf{x}_{i}+\alpha^{\prime}\right|=\left|\boldsymbol{\beta}^{t}\left(\mathbf{x}_{i}-\mathbf{x}\right)\right|=|\boldsymbol{\beta}|\left|\mathbf{x}_{i}-\mathbf{x}\right|>|\boldsymbol{\beta}| \rho$, since the distance of $\mathbf{x}_{i}$ to $L$ is greater than $\rho$.) Now $n-h_{p}+p=[(n+p) / 2] \leq h_{p}$, and (A.5) together with (A.2) yields

$$
s_{n}\left(r_{1}^{\prime}(\boldsymbol{\beta}), \ldots, r_{n}^{\prime}(\boldsymbol{\beta})\right)>\gamma\left(\rho|\boldsymbol{\beta}|-M_{Y}\right) .
$$

Combining (A.4) and (A.6) with (A.3) yields

$$
s_{n}\left(\boldsymbol{\beta}, Z^{\prime}\right)>\gamma\left(\frac{\delta}{\gamma} M^{*}+M_{Y}-M_{Y}\right)=\delta M^{*} \geq s_{n}\left(\mathbf{0}, Z^{\prime}\right),
$$


from which it follows that $\left|\operatorname{argmin}_{\boldsymbol{\beta}} s_{n}\left(\boldsymbol{\beta}, Z^{\prime}\right)\right|=\left|T\left(Z^{\prime}\right)\right|$ is bounded from above by a constant, only depending on the original sample.

\section{Appendix B Proofs and Computations of Section 3}

Lemma 3. If $K_{0}$ satisfies conditions $(G)$ and $(F)$ then $\left(F_{0, \boldsymbol{\beta}}^{+}\right)^{-1}(t)$ is strictly increasing in $|\boldsymbol{\beta}|$ for all $0 \leq t \leq 1$.

Proof : First note that since $\boldsymbol{\beta}^{t} \mathbf{x}$ depends only on $|\boldsymbol{\beta}|$, due to the sphericity of $G_{0}$, that $\left(F_{0, \boldsymbol{\beta}}^{+}\right)^{-1}(t)$ depends only on $|\boldsymbol{\beta}|$. Furthermore, $\boldsymbol{\beta}^{t} \mathbf{x}$ has the same distribution as $|\boldsymbol{\beta}| z$, where $z \sim \mathbf{c}^{t} u$ and $\mathbf{c}$ is an arbitrary unit vector. Then we have that for every $x \geq 0$,

$$
F_{0, \boldsymbol{\beta}^{+}}^{+}(x)=P\left(\left|y-\boldsymbol{\beta}^{t} u\right| \leq x\right)=E_{z}[P(|y-| \boldsymbol{\beta}|z| \leq x \mid z)]=E_{z}\left[F_{0}(x+|\boldsymbol{\beta}| z)-F_{0}(|\boldsymbol{\beta}| z-x)\right] .
$$

Since $|\boldsymbol{\beta}| \rightarrow F_{0}(|\boldsymbol{\beta}| z+x)-F_{0}(|\boldsymbol{\beta}| z-x)$ is strictly decreasing ( $F_{0}$ has a positive density) in $|\boldsymbol{\beta}|$ for every $z \neq 0$, and since $P(z=0)=0$, we have that $F_{0, \boldsymbol{\beta}}^{+}(x)$ is strictly decreasing in $|\boldsymbol{\beta}|$ for every $x \geq 0$. Therefore $\left(F_{0, \boldsymbol{\beta}}^{+}\right)^{-1}$ is strictly increasing in $|\boldsymbol{\beta}|$.

Proof of Theorem 3. Denote $c=\tilde{h}^{-1}\left(\varepsilon, s_{1}\right)$. Note that $y-\boldsymbol{\beta}^{t} \mathrm{x}$ has a symmetric and unimodal distribution at the model. Therefore the functions $g^{+}$and $g_{\boldsymbol{\beta}}^{-}$given by (3.8) and (3.9) are well-defined, even and strictly increasing on the positive numbers. (Due to the sphericity of $G_{0}$ and the fact that $G_{0}$ has no mass at zero, $\boldsymbol{\beta}^{t} u$ has no atoms.) From (3.1) and $h_{2}^{+}=\delta_{1 / 2}$ we have that $H_{\boldsymbol{\beta}}(r)=\left(F_{\boldsymbol{\beta}, r}^{+}\right)^{-1}(1 / 2)$, and we will denote $H=H_{0}$. We will use the convention that $\tilde{F}^{-1}(t)=\inf \{x ; \tilde{F}(x) \geq t\}$. Take an $\varepsilon$ between 0 and $1 / 2$.

First we will prove that $B_{\varepsilon}(T) \leq c$. Let $K=(1-\varepsilon) K_{0}+\varepsilon K^{*}$, where $K^{*}$ is an arbitrary distribution. Denote the marginals of $K$ by $G$ and $F$ and those of $K^{*}$ by $G^{*}$ and $F^{*}$. We will use the obvious notations $L_{F}, H_{F}, L_{K, \boldsymbol{\beta}}$, and $H_{K, \boldsymbol{\beta}}$. Take any $|\boldsymbol{\beta}|>c$. It suffices to prove that

$$
s(\boldsymbol{\beta}, K)>s(\mathbf{0}, K)
$$

Now

$$
H_{F}(y)=\inf \left\{t ;(1-\varepsilon)\left(F_{0}(y+t)-F_{0}(y-t)\right)+\varepsilon P_{F^{*}}(|Y-y| \leq t) \geq 1 / 2\right\} \leq g^{+}(y),
$$

from which it follows that

$$
L_{F}(y)=P_{F}\left(H_{F}(Y) \leq y\right)
$$




$$
\begin{aligned}
& \geq P_{F}\left(g^{+}(Y) \leq y\right) \\
& \geq(1-\varepsilon) P_{F_{0}}\left(g^{+}(Y) \leq y\right) \\
& =(1-\varepsilon) F_{0}^{+}\left(\left(g^{+}\right)^{-1}(y)\right),
\end{aligned}
$$

where $\left(g^{+}\right)^{-1}=\left(\left.g^{+}\right|_{[0, \infty[}\right)^{-1}$. From this it follows that

$$
\begin{aligned}
L_{F}^{-1}(t) & =\inf \left\{y ; L_{F}(y) \geq t\right\} \\
& \leq \inf \left\{y ;(1-\varepsilon) F_{0}^{+}\left(\left(g^{+}\right)^{-1}(y)\right) \geq t\right\} \\
& =g^{+}\left(\left(F_{0}^{+}\right)^{-1}\left(\frac{t}{1-\varepsilon}\right)\right) \\
& =g^{+}\left(F_{0}^{-1}\left(\frac{1+t-\varepsilon}{2(1-\varepsilon)}\right)\right) .
\end{aligned}
$$

The above inequality, combined with definition (3.12), results in

$$
s(\mathbf{0}, K) \leq s_{1}
$$

Furthermore,

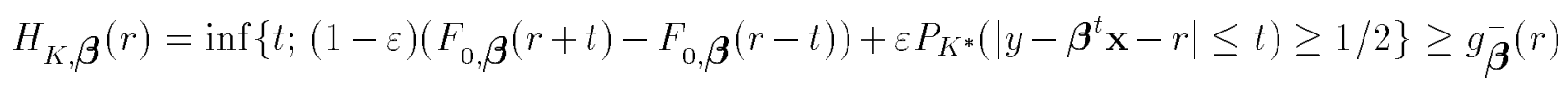

hence

$$
\begin{aligned}
L_{K, \boldsymbol{\beta}}(x) & =P_{K}\left(H_{K, \boldsymbol{\beta}}\left(y-\boldsymbol{\beta}^{t} \mathbf{x}\right) \leq x\right) \\
& \leq(1-\varepsilon) P_{K_{0}}\left(H_{K, \boldsymbol{\beta}}\left(y-\boldsymbol{\beta}^{t} \mathbf{x}\right) \leq x\right)+\varepsilon \\
& \leq(1-\varepsilon) P_{K_{0}}\left(g_{\boldsymbol{\beta}^{-}}\left(y-\boldsymbol{\beta}^{t} \mathbf{x}\right) \leq x\right)+\varepsilon \\
& =(1-\varepsilon)\left(F_{0, \boldsymbol{\beta}^{+}}^{+1}\left(\left(g_{\boldsymbol{\beta}}^{-}\right)^{-1}(x)\right)+\varepsilon \text { for } x \geq g_{\boldsymbol{\beta}^{-}}(0)\right. \\
L_{K, \boldsymbol{\beta}}(x) & =0 \text { for } x<g_{\boldsymbol{\beta}^{-}}(0)
\end{aligned}
$$

where we used $\left(g_{\boldsymbol{\beta}}^{-}\right)^{-1}=\left(\left.g_{\boldsymbol{\beta}}^{-}\right|_{[0, \infty[}\right)^{-1}$. Therefore,

$$
L_{K, \boldsymbol{\beta}}^{-1}(t) \geq \begin{cases}g_{\boldsymbol{\beta}^{-}}^{-}(0) & \text { for } t<\varepsilon \\ g_{\boldsymbol{\beta}^{-}}^{-}\left(\left(F_{0, \boldsymbol{\beta}}^{+}\right)^{-1}\left(\frac{t-\varepsilon}{1-\varepsilon}\right)\right) & \text { for } 1 \geq t \geq \varepsilon .\end{cases}
$$

Using the above inequality and $|\boldsymbol{\beta}|>c$ we get

$$
\begin{aligned}
s(\boldsymbol{\beta}, K) & =\int_{0}^{\alpha_{1}} L_{K, \boldsymbol{\beta}}^{-1}(t) h_{1}(t) d t \\
& \geq g_{\boldsymbol{\beta}}^{-}(0) \int_{0}^{\varepsilon} h_{1}(t) d t+\int_{\varepsilon}^{\alpha_{1}} h_{1}(t) g_{\boldsymbol{\beta}^{-}}\left(\left(F_{0, \boldsymbol{\beta}}^{+}\right)^{-1}\left(\frac{t-\varepsilon}{1-\varepsilon}\right)\right) d t \\
& =\tilde{h}(\varepsilon, \boldsymbol{\beta})>\tilde{h}(\varepsilon, c)=s_{1} .
\end{aligned}
$$


Inequalities (B.2) and (B.3) together imply (B.1).

In the second part of the proof we will show the other inequality $B_{\varepsilon}(T) \geq c$. Take $c_{1}<c$, a vector with $\left|\boldsymbol{\beta}^{*}\right|=c_{1}$, and consider a sequence of distributions $K_{n}=(1-\varepsilon) K_{0}+\varepsilon \Delta_{\left(\mathbf{x}_{n}, y_{n}\right)}$, with marginals $G_{n}$ and $F_{n}$, where $\mathbf{x}_{n}=\lambda_{n} \boldsymbol{\beta}^{*}, y_{n}=\mathbf{x}_{n}^{t} \boldsymbol{\beta}^{*}$, and $\lambda_{n} \rightarrow \infty$. It suffices to prove

$$
\sup _{n}\left|T\left(K_{n}\right)\right| \geq c_{1}
$$

Suppose (B.4) is not true, then there exists a subsequence $T\left(K_{n}\right)=\boldsymbol{\beta}_{n}$ such that $\lim _{n \rightarrow \infty} \boldsymbol{\beta}_{n}=\tilde{\boldsymbol{\beta}}$ with $|\tilde{\boldsymbol{\beta}}|<c_{1}$. The notations $L_{n, \boldsymbol{\beta}}$ and $H_{n, \boldsymbol{\beta}}$ are again obvious.

Since the contaminating distribution satisfies $y=\mathrm{x}^{t} \boldsymbol{\beta}^{*}$ exactly, we have

$$
\begin{aligned}
H_{n, \boldsymbol{\beta}^{*}}(r) & =\inf \left\{y ;(1-\varepsilon)\left(F_{0, \boldsymbol{\beta}^{*}}(r+y)-F_{0, \boldsymbol{\beta}^{*}}(r-y)\right)+\varepsilon I\left(\left|y_{n}-\mathbf{x}_{n}^{t} \boldsymbol{\beta}^{*}-r\right| \leq y\right) \geq 1 / 2\right\} \\
& =\inf \left\{y ;(1-\varepsilon)\left(F_{0, \boldsymbol{\beta}^{*}}(r+y)-F_{0, \boldsymbol{\beta}^{*}}(r-y)\right)+\varepsilon I(|r| \leq y) \geq 1 / 2\right\} .
\end{aligned}
$$

Note that always $H_{n, \boldsymbol{\beta}^{*}}(r) \geq|r|$ since

$$
(1-\varepsilon)\left(F_{0, \boldsymbol{\beta}^{*}}(r+|r|)-F_{0, \boldsymbol{\beta}^{*}}(r-|r|)\right)=(1-\varepsilon)\left(F_{0, \boldsymbol{\beta}^{*}}(2|r|)-\frac{1}{2}\right)<\frac{1}{2} .
$$

We have that $H_{n, \beta^{*}}(r)=|r|$ if

$$
(1-\varepsilon)\left(F_{0, \beta^{*}}(2|r|)-\frac{1}{2}\right)+\varepsilon \geq \frac{1}{2} \Leftrightarrow|r| \geq \frac{1}{2} F_{0, \beta^{*}}^{-1}\left(\frac{2-3 \varepsilon}{2(1-\varepsilon)}\right) .
$$

Therefore

$$
\begin{aligned}
H_{n, \boldsymbol{\beta}^{*}}(r) & =g_{\boldsymbol{\beta}^{*}}^{-}(r) & & \text { for }|r|<\frac{1}{2} F_{0, \boldsymbol{\beta}^{*}}^{-1}\left(\frac{2-3 \varepsilon}{2(1-\varepsilon)}\right) \\
& =|r| & & \text { for }|r| \geq \frac{1}{2} F_{0, \boldsymbol{\beta}^{*}}^{-1}\left(\frac{2-3 \varepsilon}{2(1-\varepsilon)}\right),
\end{aligned}
$$

which results in a strictly increasing and continous function. Now we want to compute

$$
\begin{aligned}
L_{n, \boldsymbol{\beta}^{*}}(x) & =(1-\varepsilon) P_{K_{0}}\left(H_{n, \boldsymbol{\beta}^{*}}\left(y-\mathbf{x}^{t} \boldsymbol{\beta}^{*}\right) \leq x\right)+\varepsilon I\left(H_{n, \boldsymbol{\beta}^{*}}(0) \leq x\right) \\
& =(1-\varepsilon) P_{K_{0}}\left(\left|y-\mathbf{x}^{t} \boldsymbol{\beta}^{\star}\right| \leq H_{n, \boldsymbol{\beta}^{*}}^{-1}(x)\right)+\varepsilon I\left(g_{\boldsymbol{\beta}^{*}}^{-}(0) \leq x\right) .
\end{aligned}
$$

With the aid of (B.5), this results in

$$
L_{n, \boldsymbol{\beta}^{*}}(x)= \begin{cases}0 & \text { for } x<g_{\boldsymbol{\beta}^{*}}^{-}(0) \\ (1-\varepsilon) F_{0, \boldsymbol{\beta}^{*}}^{+}\left(\left(g_{\boldsymbol{\beta}^{*}}^{-}\right)^{-1}(x)\right)+\varepsilon & \text { for } g_{\boldsymbol{\beta}^{*}}^{-}(0) \leq x<\frac{1}{2} F_{0, \boldsymbol{\beta}^{*}}^{-1}\left(\frac{2-3 \varepsilon}{2(1-\varepsilon)}\right) .\end{cases}
$$

Now we note that

$$
\lim _{t \uparrow \frac{1}{2} F_{0, \beta^{*}}^{-1}\left(\frac{2-3 \varepsilon}{2(1-\varepsilon)}\right)} L_{n, \boldsymbol{\beta}^{*}}(t)=(1-\varepsilon) F_{0, \beta^{*}}^{+}\left(\frac{1}{2} F_{0, \beta^{*}}^{-1}\left(\frac{2-3 \varepsilon}{2(1-\varepsilon)}\right)\right)+\varepsilon>\frac{1}{2}
$$




$$
\Leftrightarrow 2 F_{0, \boldsymbol{\beta}^{*}}^{-1}\left(\frac{3-4 \varepsilon}{4(1-\varepsilon)}<F_{0, \boldsymbol{\beta}^{*}}^{-1}\left(\frac{2-3 \varepsilon}{2(1-\varepsilon)}\right),\right.
$$

and this last inequality is true for every symmetric unimodal distribution. Therefore we may conclude from (B.6) that

$$
L_{n, \boldsymbol{\beta}^{*}}^{-1}(t)= \begin{cases}g_{\boldsymbol{\beta}^{*}}^{-}(0) & \text { for } 0<t<\varepsilon \\ g_{\boldsymbol{\beta}^{*}}^{-}\left(\left(F_{0, \boldsymbol{\beta}^{*}}^{+}\right)^{-1}\left(\frac{t-\varepsilon}{1-\varepsilon}\right)\right) & \text { for } \frac{1}{2} \geq \alpha_{1} \geq t \geq \varepsilon .\end{cases}
$$

From (B.7) it follows that

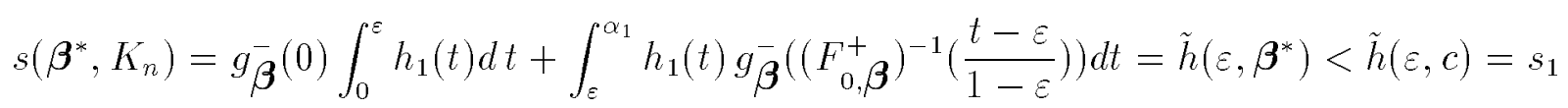

since $\left|\boldsymbol{\beta}^{*}\right|<c$.

Now denote $x_{n}=\left(y_{n}-\boldsymbol{\beta}_{n}^{t} \mathbf{x}_{n}\right)$. Then $\left|x_{n}\right|=\left.\lambda_{n}|| \boldsymbol{\beta}^{*}\right|^{2}-\boldsymbol{\beta}_{n}^{t} \boldsymbol{\beta}^{*} \mid \rightarrow \infty$, since $\left|\boldsymbol{\beta}_{n}\right| \rightarrow|\tilde{\boldsymbol{\beta}}|<$ $\left|\boldsymbol{\beta}^{\star}\right|$. Suppose w.1.o.g. that $x_{n}>0$. We have

$$
H_{n, \boldsymbol{\beta}_{n}}(r)=\inf \left\{y ;(1-\varepsilon)\left(F_{0, \boldsymbol{\beta}_{n}}(r+y)-F_{0, \boldsymbol{\beta}_{n}}(r-y)\right)+\varepsilon I\left(\left|x_{n}-r\right| \leq y\right) \geq 1 / 2\right\}
$$

We introduce the function $g_{\boldsymbol{\beta}}^{+}(y)$ which is defined as the solution of the equation

$$
F_{\boldsymbol{\beta}}\left(y+g_{\boldsymbol{\beta}}^{+}(y)\right)-F_{0}\left(y-g_{\boldsymbol{\beta}}^{+}(y)\right)=\frac{1}{2(1-\varepsilon)} .
$$

Note that $g^{+}=g_{0}^{+}$. Denote by $r_{n}$ the unique solution of the equation

$$
\left|x_{n}-r\right|=g_{\boldsymbol{\beta}_{n}^{+}}^{+}\left(x_{n}\right) \text {. }
$$

For $n$ large enough, $r_{n}$ will be positive. Then it follows from (B.9) that

$$
\begin{array}{ll}
H_{n, \boldsymbol{\beta}_{n}}(r)=g_{\boldsymbol{\beta}_{n}}^{+}(r) & \text { for } r<r_{n} \\
H_{n, \boldsymbol{\beta}_{n}}(r)=g_{\boldsymbol{\beta}_{n}}^{-}(r) & \text { for } r \geq r_{n} .
\end{array}
$$

$\mathrm{On}]-\infty, r_{n}$ [ this function is even, and it is increasing on $\left[0, r_{n}\left[\right.\right.$. At $r_{n}$ it makes a small jump downwards and then it increases again. Now we want to compute

$$
L_{n, \boldsymbol{\beta}_{n}}(x)=(1-\varepsilon) P_{K_{0}}\left(H_{n, \boldsymbol{\beta}_{n}}\left(y-\mathbf{x}^{t} \boldsymbol{\beta}^{*}\right) \leq x\right)+\varepsilon I\left(H_{n, \boldsymbol{\beta}_{n}}\left(x_{n}\right) \leq x\right) .
$$

Denote by $r_{n}^{\prime}$ the solution of $g_{\boldsymbol{\beta}_{n}}^{+}\left(r_{n}^{\prime}\right)=g_{\boldsymbol{\beta}_{n}}^{-}\left(r_{n}\right)$. Note that $H_{n, \boldsymbol{\beta}_{n}}\left(x_{n}\right) \rightarrow \infty$ yields

$$
L_{n, \boldsymbol{\beta}_{n}}(x)=(1-\varepsilon) F_{0, \boldsymbol{\beta}_{n}}^{+}\left(\left(g_{\boldsymbol{\beta}_{n}}^{+}\right)^{-1}(x)\right) \text { for } x \leq r_{n}^{\prime}
$$


for $n$ large enough, since $r_{n} \rightarrow \infty$. Putting $t_{n}=L_{n, \boldsymbol{\beta}_{n}}\left(r_{n}^{\prime}\right)=(1-\varepsilon) F_{0, \boldsymbol{\beta}_{n}}^{+}\left(g_{\boldsymbol{\beta}_{n}^{-}}\left(r_{n}\right)\right)$, it then follows from (B.11) that

$$
L_{n, \boldsymbol{\beta}_{n}}^{-1}(t)=g_{\boldsymbol{\beta}_{n}}^{+}\left(\left(F_{0, \beta_{n}}^{+}\right)^{-1}\left(\frac{t}{1-\varepsilon}\right)\right) \text { for } t \leq t_{n} .
$$

From condition (GF) it follows that $\boldsymbol{\beta} \rightarrow g_{\boldsymbol{\beta}}^{+}\left(\left(F_{0, \boldsymbol{\beta}}^{+}\right)^{-1}(t /(1-\varepsilon))\right)$ is increasing in $|\boldsymbol{\beta}|$. Since $t_{n} \rightarrow 1-\varepsilon$ (because $\boldsymbol{\beta}_{n}$ is bounded and $\left.r_{n} \rightarrow \infty\right)$ and $(1-\varepsilon)>\alpha_{1}$ we get, using (B.12),

$$
s\left(\boldsymbol{\beta}_{n}, K_{n}\right) \geq \int_{0}^{\alpha_{1}} g^{+}\left(\left(F_{0}^{+}\right)^{-1}\left(\frac{t}{1-\varepsilon}\right)\right) h_{1}(t) d t=s_{1} .
$$

Inequalities (B.8) and (B.13) are in contradiction with $\boldsymbol{\beta}_{n}=\operatorname{argmin} \boldsymbol{\beta} s(\boldsymbol{\beta}, K)$, therefore (B.4) must be true.

Finally, we will show that

$$
\lim _{\varepsilon \uparrow \frac{1}{2}}\left|B_{\varepsilon}(T)\right|=\infty
$$

Definitions (3.8) and (3.9) imply that

$$
\lim _{\varepsilon \uparrow \frac{1}{2}} g^{+}(r)=0 \text { and } \lim _{\varepsilon \uparrow \frac{1}{2}} g_{\boldsymbol{\beta}^{-}}^{-}(r)=0
$$

from which it follows that $\lim _{\varepsilon \uparrow \frac{1}{2}} s_{1}=\infty$ and $\lim _{\varepsilon \uparrow \frac{1}{2}} \tilde{h}(\varepsilon, \boldsymbol{\beta})=0$ for every finite $|\boldsymbol{\beta}|$. Therefore (B.14) must be true.

Derivation of equations $\mathbf{( 3 . 1 3 )}$ and (3.14). If $(\mathrm{x}, y) \sim N\left(\mathbf{0}, \mathrm{I}_{p+1}\right)$ then $y-\boldsymbol{\beta}^{t} \mathrm{x}$ has the same distribution as $z \sqrt{1+|\boldsymbol{\beta}|^{2}}$, where $z$ has a univariate standard normal distribution. From this one can easily see that

$$
\left(\Phi_{\boldsymbol{\beta}}^{+}\right)^{-1}(s)=\sqrt{1+|\boldsymbol{\beta}|^{2}} \Phi^{-1}\left(\frac{1+s}{2}\right) \text { and } g_{\boldsymbol{\beta}^{-}}^{-}(r)=\sqrt{1+|\boldsymbol{\beta}|^{2}} g^{-}\left(\frac{r}{\sqrt{1+|\boldsymbol{\beta}|^{2}}}\right)
$$

and that condition (GF) is satisfied. Using these equations we get

$$
g_{\boldsymbol{\beta}^{-}}^{-}(0)=\sqrt{1+|\boldsymbol{\beta}|^{2}} g^{-}(0)=\sqrt{1+|\boldsymbol{\beta}|^{2}} \Phi^{-1}\left(\frac{3-4 \varepsilon}{4(1-\varepsilon)}\right)
$$

and

$$
g_{\boldsymbol{\beta}}^{-}\left(\left(\Phi_{\boldsymbol{\beta}}^{+}\right)^{-1}\left(\frac{t-\varepsilon}{1-\varepsilon}\right)\right)=\sqrt{1+|\boldsymbol{\beta}|^{2}} g^{-}\left(\frac{1+t-2 \varepsilon}{2(1-\varepsilon)}\right) .
$$

Using $\tilde{h}\left(\varepsilon, B_{\varepsilon}(T)\right)=s_{1}$ and equations (3.10) and (3.12) it then follows that

$$
B_{\varepsilon}(T)^{2}=\left\{\frac{\int_{0}^{\alpha_{1}} h_{1}(t) g^{+}\left(\Phi^{-1}\left(\frac{1+t-\varepsilon}{2(1-\varepsilon)}\right)\right) d t}{\Phi^{-1}\left(\frac{3-4 \varepsilon}{4(1-\varepsilon)}\right) \int_{0}^{\varepsilon} h_{1}(t) d t+\int_{\varepsilon}^{\alpha_{1}} h_{1}(t) g^{-}\left(\Phi^{-1}\left(\frac{1+t-2 \varepsilon}{2(1-\varepsilon)}\right)\right) d t}\right\}^{2}-1
$$


or, using the appropriate change in variables,

$$
B_{\varepsilon}(T)^{2}=\left\{\frac{\int_{0}^{\Phi^{-1}\left(\frac{1+\alpha_{1}-\varepsilon}{2(1-\varepsilon)}\right)} h_{1}\left((1-\varepsilon)(2 \Phi(r)-1) g^{+}(r) d \Phi(r)\right.}{\Phi^{-1}\left(\frac{3-4 \varepsilon}{4(1-\varepsilon)}\right) \frac{\int_{0}^{\varepsilon} h_{1}(t) d t}{2(1-\varepsilon)}+\int_{0}^{\Phi^{-1}\left(\frac{1+\alpha_{1}-\varepsilon}{2(1-\varepsilon)}\right)} h_{1}((1-\varepsilon)(2 \Phi(r)-1)+\varepsilon) g^{-}(r) d \Phi(r)}\right\}^{2}-1 .
$$

From (B.16) we obtain (3.13) by taking $h_{1}(t)=I\left(t \leq \alpha_{1}\right)$, and from (B.15) with $h_{1}=\delta_{\alpha_{1}}$ formula (3.14) readily follows.

\section{Maxbias curves for Signed R-Estimators:}

Proof of equation (3.16): Denote $c=\tilde{l}^{-1}(1-\varepsilon, \varepsilon, \tilde{l}(1-\varepsilon, 0,0))$ and $s_{1}=\tilde{l}(1-\varepsilon, 0,0)$, and take $\varepsilon<\min (\alpha, 1-\alpha)$.

First we will prove that $B_{\varepsilon}(T) \leq c$. Let $K=(1-\varepsilon) K_{0}+\varepsilon K^{*}$, where $K^{*}$ is an arbitrary distribution. Denote the marginals of $K$ by $G$ and $F$. Take any $|\boldsymbol{\beta}|>c$. It suffices to prove that

$$
s(\boldsymbol{\beta}, K)>s(\mathbf{0}, K)
$$

We have

$$
\begin{aligned}
s(\mathbf{0}, K) & =\int_{0}^{\alpha} h^{+}(s)\left(\left(F^{+}\right)^{-1}(s)\right)^{k} d s \\
& \leq \int_{0}^{1-\varepsilon} h^{+}(s)\left(\left(F_{0}^{+}\right)^{-1}\left(\frac{s}{1-\varepsilon}\right)\right)^{k} d s \\
& =\tilde{l}(1-\varepsilon, 0,0)=s_{1}
\end{aligned}
$$

since $1-\varepsilon>\alpha$. Furthermore

$$
\begin{aligned}
s(\boldsymbol{\beta}, K) & \geq \int_{\varepsilon}^{\alpha} h^{+}(s)\left(\left(F_{\boldsymbol{\beta}}^{+}\right)^{-1}(s)\right)^{k} d s \\
& \geq \int_{\varepsilon}^{\alpha} h^{+}(s)\left(\left(F_{0, \boldsymbol{\beta}}^{+}\right)^{-1}\left(\frac{s-\varepsilon}{1-\varepsilon}\right)\right)^{k} d s \\
& \geq \tilde{l}(1-\varepsilon, \varepsilon,|\boldsymbol{\beta}|) \\
& >\tilde{l}(1-\varepsilon, \varepsilon, c)=s_{1},
\end{aligned}
$$

where we used $|\boldsymbol{\beta}|>c$ and Lemma 3 for the last inequalities. Combining (B.19) and (B.18) gives (B.17).

Now we will prove the other inequality, $B_{\varepsilon}(T) \geq c$. Take any $c_{1}<c$ and a vector $\boldsymbol{\beta}^{\star}$ with $\left|\boldsymbol{\beta}^{*}\right|=c_{1}$. Consider the following sequence of contaminating distributions: $K_{n}=$ 
$(1-\varepsilon) K_{0}+\varepsilon \Delta_{\mathbf{x}_{n}, y_{n}}$, where $\mathbf{x}_{n}=\lambda_{n} \boldsymbol{\beta}^{*}, y_{n}=\mathbf{x}_{n}^{t} \boldsymbol{\beta}^{*}$, and $\lambda_{n} \rightarrow \infty$. Denote the marginals of $K_{n}$ by $G_{n}$ and $F_{n}$. It is sufficient to prove that

$$
\sup _{n}\left|T\left(K_{n}\right)\right| \geq c_{1}
$$

Suppose that (B.20) is not true. Then we can find a subsequence $\boldsymbol{\beta}_{n}$ such that $T\left(K_{n}\right)=\boldsymbol{\beta}_{n}$, where $\boldsymbol{\beta}_{n} \rightarrow \tilde{\boldsymbol{\beta}}$ with $|\tilde{\boldsymbol{\beta}}|<c_{1}$. We have that

$$
\begin{aligned}
s\left(\boldsymbol{\beta}^{*}, K_{n}\right) & =\int_{0}^{\alpha} h^{+}(s)\left(\left(F_{n, \beta^{*}}^{+}\right)^{-1}(s)\right)^{k} d s \\
& =\int_{\varepsilon}^{\alpha} h^{+}(s)\left(\left(F_{0, \beta^{*}}^{+}\right)^{-1}\left(\frac{s-\varepsilon}{1-\varepsilon}\right)\right)^{k} d s \\
& =\tilde{l}\left(1-\varepsilon, \varepsilon,\left|\boldsymbol{\beta}^{*}\right|\right)<s_{1},
\end{aligned}
$$

where we used for the last equality that $\left|\boldsymbol{\beta}^{*}\right|<c$. Now define $x_{n}=\left|y_{n}-\mathbf{x}_{n}^{t} \boldsymbol{\beta}_{n}\right|=$ $\lambda_{n}\left(\left|\boldsymbol{\beta}^{*}\right|^{2}-\boldsymbol{\beta}_{n}^{t} \boldsymbol{\beta}^{*}\right)$, which converges to infinity (using the fact that $|\tilde{\boldsymbol{\beta}}|<\left|\boldsymbol{\beta}^{*}\right|$.) Then $z_{n}=F_{0, \boldsymbol{\beta}_{n}}^{+}\left(x_{n}\right)$ tends to one. We have that

$$
\begin{aligned}
s\left(\boldsymbol{\beta}_{n}, K_{n}\right) & =\int_{0}^{\alpha} h^{+}(s)\left(\left(F_{n, \boldsymbol{\beta}_{n}}^{+}\right)^{-1}(s)\right)^{k} d s \\
& \geq \int_{0}^{\min \left(z_{n}(1-\varepsilon), \alpha\right)} h^{+}(s)\left(\left(F_{0, \boldsymbol{\beta}_{n}}^{+}\right)^{-1}(s /(1-\varepsilon))\right)^{k} d s \\
& \rightarrow \tilde{l}(1-\varepsilon, 0,0)=s_{1},
\end{aligned}
$$

where we used Lemma 3 and $1-\varepsilon>\alpha$. Equations (B.21) and (B.22) are in contradiction with the definition of $\boldsymbol{\beta}_{n}=\operatorname{argmin}_{\boldsymbol{\beta}} s\left(\boldsymbol{\beta}, K_{n}\right)$, and therefore (B.20) has to be true.

Now take an $\varepsilon \geq \alpha$. Then $s\left(\boldsymbol{\beta}, K_{n}\right)=0$ due to the second line of (B.21), for any choice of $\boldsymbol{\beta}$. In particular, we can let $|\boldsymbol{\beta}|$ tend to infinity, which yields $B_{\varepsilon}(T)=\infty$.

For an $\varepsilon>1-\alpha$ (and $\varepsilon<\alpha$ w.l.o.g), take an arbitrary $\boldsymbol{\beta}^{*}$ and again construct a distribution $K_{n}$ as before. From (B.21) we have that

$$
s\left(\boldsymbol{\beta}^{*}, K_{n}\right)=\tilde{l}\left(1-\varepsilon, \varepsilon, \boldsymbol{\beta}^{*}\right)<\infty .
$$

Take any $|\boldsymbol{\beta}|<\left|\boldsymbol{\beta}^{*}\right|$ and put $z_{n}=F_{0, \boldsymbol{\beta}^{(}}^{+}\left(x_{n}\right)$ where $x_{n}=\left|y_{n}-u_{n}^{t} \boldsymbol{\beta}\right|$ again tends to infinity. Then $z_{n}$ will be smaller than $\alpha /(1-\varepsilon)$ for large $n$, hence

$$
s\left(\boldsymbol{\beta}, K_{n}\right) \geq x_{n} \int_{z_{n}(1-\varepsilon)}^{\alpha} h^{+}(t) d t \rightarrow \infty .
$$

From this it follows that $\left|T\left(K_{n}\right)\right| \geq\left|\boldsymbol{\beta}^{*}\right|$. Since $\boldsymbol{\beta}^{*}$ was chosen arbitrarily, it holds that $B_{\varepsilon}(T)=\infty$. 
Remark: Note that if $\alpha>1 / 2$ and $h$ does not contain $\delta_{\alpha}$, then

$$
\lim _{\varepsilon \uparrow 1-\alpha} B_{\varepsilon}(T)<\infty
$$

which is quite remarkable. Thus it is possible that the maxbias curve has a discontinuity at its breakdown point. What happens in $\varepsilon=1-\alpha$ depends on whether $h^{+}(\alpha)>0$ (then the bias curve explodes at $1-\alpha$ ) or $h^{+}(\alpha)=0$ (in which case the bias curve is finite in $1-\alpha$ ). If $\alpha \leq 1 / 2$ it remains true that $\lim _{\varepsilon \uparrow \alpha} B_{\varepsilon}(T)=\infty$.

\section{Appendix C Proofs of Sections 4 and 6}

Lemma 4. Suppose that a regression functional $T(K)$ is defined for every distribution $K$ of carriers and responses, and satisfies

$$
\int \psi\left(y-\boldsymbol{\beta}^{t} \mathbf{x}, \boldsymbol{\beta}, F_{\boldsymbol{\beta}}\right) \mathbf{x} d K(\mathbf{x}, y)=0
$$

where the carriers $\mathrm{x}$ have distribution $G$, the responses $y$ have distribution $F$ and $F_{\boldsymbol{\beta}}$ is the distribution of $y-\boldsymbol{\beta}^{t} \mathrm{x}$. Then the influence function at the model distribution $K_{0}=\left(G_{0}, F_{0}\right)$, where $T\left(K_{0}\right)=0$ and $E_{G_{0}}[\mathbf{x}]=0$, is given by

$$
I F\left(\mathrm{x}_{0}, y_{0}\right)=\psi_{0}\left(y_{0}\right) \Sigma^{-1} \mathbf{x}_{0}
$$

where $\boldsymbol{\Sigma}=E_{G_{0}}\left[\mathbf{x x}^{t}\right]$ and $\psi_{0}(y)=\psi\left(y, \mathbf{0}, F_{0}\right)$.

Proof. Consider the distribution $K_{\varepsilon}=(1-\varepsilon) K_{0}+\varepsilon \Delta_{\left(\mathrm{x}_{0}, y_{0}\right)}$ and denote its marginals by $G_{\varepsilon}$ and $F_{\varepsilon}$. Denote $\boldsymbol{\beta}_{\varepsilon}=T\left(K_{\varepsilon}\right)$. Then definition (4.1) can be written as

$$
\left.\frac{\partial \boldsymbol{\beta}_{\varepsilon}}{\partial \varepsilon}\right|_{\varepsilon=0}=I F\left(\mathbf{x}_{0}, y_{0}\right)
$$

The necessary condition (4.2) on $\boldsymbol{\beta}_{\varepsilon}$ becomes

$$
(1-\varepsilon) \int \psi\left(y-\boldsymbol{\beta}_{\varepsilon}^{t} \mathbf{x}, \boldsymbol{\beta}_{\varepsilon},\left(F_{\varepsilon}\right)_{\boldsymbol{\beta}_{\varepsilon}}\right) \mathbf{x} d K_{0}(\mathbf{x}, y)+\varepsilon \psi\left(y_{0}-\boldsymbol{\beta}_{\varepsilon}^{t} \mathbf{x}_{0}, \boldsymbol{\beta}_{\varepsilon},\left(F_{\varepsilon}\right)_{\boldsymbol{\beta}_{\varepsilon}}\right) \mathbf{x}_{0}=0
$$

Differentiating the above equation and evaluating in $\varepsilon=0$ yields

$$
0+\left.\int \frac{\partial \psi\left(y-\boldsymbol{\beta}_{\varepsilon}^{t} \mathbf{x}, \boldsymbol{\beta}_{\varepsilon},\left(F_{\varepsilon}\right)_{\boldsymbol{\beta}_{\varepsilon}}\right)}{\partial \varepsilon}\right|_{\varepsilon=0} \mathbf{x} d K_{0}(\mathbf{x}, y)+\psi_{0}\left(y_{0}\right) \mathbf{x}_{0}=0
$$

Now

$$
\left.\frac{\partial \psi\left(y-\boldsymbol{\beta}_{\varepsilon}^{t} \mathbf{x}, \boldsymbol{\beta}_{\varepsilon},\left(F_{\varepsilon}\right)_{\boldsymbol{\beta}_{\varepsilon}}\right)}{\partial \varepsilon}\right|_{\varepsilon=0}(x)=-\frac{\partial}{\partial y} \psi\left(y, \mathbf{0}, F_{0}\right)\left(\left.\frac{\partial \boldsymbol{\beta}_{\varepsilon}}{\partial \varepsilon}\right|_{\varepsilon=0}\right)^{t} \mathbf{x}
$$




$$
+\left.\left(\left.\frac{\partial}{\partial \boldsymbol{\beta}} \psi\left(y, \boldsymbol{\beta}, F_{0}\right)\right|_{\boldsymbol{\beta}_{=\mathbf{0}}}\right)^{t} \frac{\partial \boldsymbol{\beta}}{\partial \varepsilon}\right|_{\varepsilon=0}+\left.\frac{\partial \psi\left(y, \mathbf{0}, F_{\varepsilon, \boldsymbol{\beta}_{\varepsilon}}\right)}{\partial \varepsilon}\right|_{\varepsilon=0}
$$

The second term is independent of $\mathrm{x}$, so it will vanish in the integration of formula (C.3) since $E_{G_{0}}[\mathbf{x}]=0$. This is also the case for the third term, since

$$
\begin{aligned}
& \left.\frac{\partial\left(F_{\varepsilon}\right) \boldsymbol{\beta}_{\varepsilon}(x)}{\partial \varepsilon}\right|_{\varepsilon=0} \\
& \quad=\left.\frac{\partial\left[(1-\varepsilon) E_{G_{0}}\left[F_{0}\left(x+\boldsymbol{\beta}_{\varepsilon}^{t} \mathbf{x}\right)\right]+\varepsilon I\left(y_{0} \leq x_{0}+\boldsymbol{\beta}_{\varepsilon}^{t} \mathbf{x}\right)\right]}{\partial \varepsilon}\right|_{\varepsilon=0} \\
& =-F_{0}(x)+\left(\left.\frac{\partial \boldsymbol{\beta}_{\varepsilon}}{\partial \varepsilon}\right|_{\varepsilon=0}\right)^{t} E_{G_{0}}[\mathbf{x}] f_{0}(x)+I\left(y_{0} \leq x\right)=I\left(y_{0} \leq x\right)-F_{0}(x) .
\end{aligned}
$$

Therefore, (C.3) results in

$$
-\left.\int \psi_{0}^{\prime}(y) \mathbf{x x}^{t} \frac{\partial \boldsymbol{\beta}_{\varepsilon}}{\partial \varepsilon}\right|_{\varepsilon=0} d G_{0}(\mathbf{x}) d F_{0}(y)+\psi_{0}\left(y_{0}\right) \mathbf{x}_{0}=0
$$

which yields (C.2).

Derivation of equation (4.2). If we use the symbol $R^{+}(\cdot)$ to indicate the signed rank statistic, (1.11) and (3.2) correspond with

$$
s_{\mathrm{NL}}=\sum_{i=1}^{n} h_{1}\left(R^{+}\left(H\left(r_{i}(\boldsymbol{\beta})\right)\right)\right) H\left(r_{i}(\boldsymbol{\beta})\right) .
$$

Similarly, (1.12) and (3.1) correspond with

$$
H\left(r_{i}(\boldsymbol{\beta})\right)=\sum_{j=1}^{n} h_{2}^{+}\left(R^{+}\left(r_{i}(\boldsymbol{\beta})-r_{j}(\boldsymbol{\beta})\right)\right)\left|r_{i}(\boldsymbol{\beta})-r_{j}(\boldsymbol{\beta})\right| .
$$

Differentiating (C.5) gives

$$
\frac{\partial}{\partial \boldsymbol{\beta}} H\left(r_{i}(\boldsymbol{\beta})\right)=\sum_{j=1}^{n} h_{2}^{+}\left(R^{+}\left(r_{i}(\boldsymbol{\beta})-r_{j}(\boldsymbol{\beta})\right)\right) \operatorname{sign}\left(r_{i}(\boldsymbol{\beta})-r_{j}(\boldsymbol{\beta})\right)\left(\mathbf{x}_{j}-\mathbf{x}_{i}\right)
$$

Computing the derivative of (C.4) and plugging in (C.6) yields

$$
\begin{aligned}
\frac{\partial}{\partial \boldsymbol{\beta}} s_{\mathrm{NL}}(\boldsymbol{\beta}) & =\sum_{i=1}^{n} h_{1}\left(R^{+}\left(H\left(r_{i}(\boldsymbol{\beta})\right)\right) \frac{\partial}{\partial \boldsymbol{\beta}} H\left(r_{i}(\boldsymbol{\beta})\right)\right. \\
& =-\sum_{i=1}^{n} \sum_{j=1}^{n} h_{1}\left(R^{+}\left(H\left(r_{i}(\boldsymbol{\beta})\right)\right)\right) h_{2}^{+}\left(R^{+}\left(r_{i}(\boldsymbol{\beta})-r_{j}(\boldsymbol{\beta})\right)\right) \operatorname{sign}\left(r_{i}(\boldsymbol{\beta})-r_{j}(\boldsymbol{\beta})\right) \mathbf{x}_{i} \\
& +\sum_{i=1}^{n} \sum_{j=1}^{n} h_{1}\left(R^{+}\left(H\left(r_{j}(\boldsymbol{\beta})\right)\right)\right) h_{2}^{+}\left(R^{+}\left(r_{j}(\boldsymbol{\beta})-r_{i}(\boldsymbol{\beta})\right)\right) \operatorname{sign}\left(r_{j}(\boldsymbol{\beta})-r_{i}(\boldsymbol{\beta})\right) \mathbf{x}_{i}
\end{aligned}
$$


Setting the above expression equal to zero yields

$$
\begin{array}{r}
\sum_{i=1}^{n}\left[\sum_{j=1}^{n} h_{1}\left(R^{+}\left(H\left(r_{j}(\boldsymbol{\beta})\right)\right)\right) h_{2}^{+}\left(R^{+}\left(r_{i}(\boldsymbol{\beta})-r_{j}(\boldsymbol{\beta})\right)\right) \operatorname{sign}\left(r_{i}(\boldsymbol{\beta})-r_{j}(\boldsymbol{\beta})\right)\right. \\
\left.-\sum_{j=1}^{n} h_{1}\left(R^{+}\left(H\left(r_{i}(\boldsymbol{\beta})\right)\right)\right) h_{2}^{+}\left(R^{+}\left(r_{j}(\boldsymbol{\beta})-r_{i}(\boldsymbol{\beta})\right)\right) \operatorname{sign}\left(r_{j}(\boldsymbol{\beta})-r_{i}(\boldsymbol{\beta})\right)\right] \mathbf{x}_{i}=0
\end{array}
$$

Then, using definitions (4.3) and (4.4), and the notations $H_{\boldsymbol{\beta}}, L_{\boldsymbol{\beta}}$, and $F_{\boldsymbol{\beta}, r}^{+}$of Section 4, one can see that the functional version of (C.7) is given by (4.2).

Derivation of equations (4.8) and (4.9). Filling in $\boldsymbol{\beta}=\mathbf{0}$ in (4.4) gives

$$
\begin{aligned}
\tilde{\mu}(r, s, \mathbf{0}) & =h_{2}^{+}\left(F_{0, r}^{+}(s-r)\right) & & \text { for } r<s \\
& =-h_{2}^{+}\left(F_{0, r}^{+}(r-s)\right) & & \text { for } r>s .
\end{aligned}
$$

Using $F_{0, r}^{+}(x)=F_{0}(r+x)-F_{0}(r-x)$ then yields

$$
\begin{aligned}
\tilde{\mu}(r, s, \mathbf{0}) & =h_{2}^{+}\left(F_{0}(s)-F_{0}(2 r-s)\right) & & \text { for } r<s \\
& =-h_{2}^{+}\left(F_{0}(2 r-s)-F_{0}(s)\right) & & \text { for } r>s .
\end{aligned}
$$

Using the definition of $G_{r}(s)=\left(1+F_{0}(s)-F(2 r-s)\right) / 2$ results in

$$
\begin{aligned}
\tilde{\mu}(r, s, \mathbf{0}) & =h_{2}^{+}\left(2 G_{r}(s)-1\right) & & \text { for } s>r \\
& =-h_{2}^{+}\left(1-2 G_{r}(s)\right) & & \text { for } s<r .
\end{aligned}
$$

Finally, from definition (4.6) and the fact that $G_{r}(r)=1 / 2$ we obtain

$$
\tilde{\mu}(r, s, \mathbf{0})=h_{2}\left(G_{r}(s)\right)
$$

from which (4.8) readily follows.

To prove equation (4.9), note that putting $\boldsymbol{\beta}=\mathbf{0}$ in (3.1) results in

$$
\begin{aligned}
H(y) & =H_{\mathbf{0}}(y) \\
& =\int h_{2}^{+}\left(F_{0, y}^{+}\left(y_{1}\right)\right) y_{1} d F_{0, y}^{+}\left(y_{1}\right) \\
& =\int h_{2}^{+}\left(F_{0, y}^{+}\left(\left|y_{1}-y\right|\right)\right)\left|y_{1}-y\right| d F_{0}\left(y_{1}\right) \\
& =\int \tilde{\mu}\left(y, y_{1}, \mathbf{0}\right)\left(y_{1}-y\right) d F_{0}\left(y_{1}\right)
\end{aligned}
$$

where we used definition (4.4). Using (C.10) then results in (4.9).

Derivation of equations (4.10) and (4.11). We will derive (4.10) from equation (4.9), thereby using delta functions. Denote the solution of the equation $F_{0}(y+z)-F_{0}(y-$ 
$z)=1 / 2$ by $\tilde{H}(y)$. From condition $(\mathrm{F})$ it follows that $\tilde{H}(z)$ is unique, and forms an even function which is strictly increasing on the positive numbers. We need to show that $\tilde{H}=H$. From definition (4.7) it easily follows that

$$
G_{y}\left(y_{1}\right)=3 / 4 \Leftrightarrow y_{1}=y+\tilde{H}(y) \text { and } G_{y}\left(y_{1}\right)=1 / 4 \Leftrightarrow y_{1}=y-\tilde{H}(y)
$$

We also have

$$
\frac{d}{d y_{1}} G_{y}\left(y_{1}\right)=\frac{f\left(y_{1}\right)+f\left(2 y-y_{1}\right)}{2}>0
$$

From the above equations it follows that

$$
\begin{aligned}
H(y) & =\frac{1}{2} \int\left(\delta_{3 / 4}\left(\left(G_{y}\left(y_{1}\right)\right)+\delta_{1 / 4}\left(G_{y}\left(y_{1}\right)\right)\right)\left(y_{1}-y\right) f_{0}\left(y_{1}\right) d y_{1}\right. \\
& =\frac{1}{2}\left\{\left.\frac{\left(y_{1}-y\right) f_{0}\left(y_{1}\right)}{\frac{d}{d y_{1}} G_{y}\left(y_{1}\right)}\right|_{y_{1}=G_{y}^{-1}(3 / 4)}-\left.\frac{\left(y_{1}-y\right) f_{0}\left(y_{1}\right)}{\frac{d}{d y_{1}} G_{y}\left(y_{1}\right)}\right|_{y_{1}=G_{y}^{-1}(1 / 4)}\right\} \\
& =\frac{1}{2}\left\{\frac{2 \tilde{H}(y) f_{0}(y+\tilde{H}(y))}{f_{0}(y+\tilde{H}(y))+f_{0}(y-\tilde{H}(y))}-\frac{-2 \tilde{H}(y) f_{0}(y-\tilde{H}(y))}{f_{0}(y-\tilde{H}(y))+f_{0}(y+\tilde{H}(y))}\right\} \\
& =\tilde{H}(y),
\end{aligned}
$$

which proves $(4.10)$.

In order to obtain (4.11), let us first compute the second term of (4.8):

$$
\begin{aligned}
-I & {[L(H(y)) \leq 1 / 2] \frac{1}{2} \int\left(\delta_{3 / 4}-\delta_{1 / 4}\right)\left(G_{y}\left(y_{1}\right)\right) f_{0}\left(y_{1}\right) d y_{1} } \\
& =I\left(|y| \leq F_{0}^{-1}(3 / 4)\right) \frac{f_{0}(y-H(y))-f_{0}(y+H(y))}{f_{0}(y+H(y))+f_{0}(y-H(y))} \\
& =I(|y| \leq q) H^{\prime}(y),
\end{aligned}
$$

where we used (4.10) to compute the derivative of $H$. Now, using that $L(H(y)) \leq 1 / 2$ if and only if $|y| \leq q$, the first term of (4.8) becomes

$$
\int_{-q}^{q} h_{2}\left(G_{z}(y)\right) f_{0}(z) d z
$$

Introducing two functions $v(z)=z+H(z)$ and $u(z)=z-H(z)$, which are both increasing since $\left|H^{\prime}(z)\right|<1$, we obtain

$G_{y}(z)=3 / 4 \Leftrightarrow y=z+H(z) \Leftrightarrow z=v^{-1}(y)$ and $G_{y}(z)=1 / 4 \Leftrightarrow y=z-H(z) \Leftrightarrow z=u^{-1}(y)$.

Furthermore,

$$
\frac{d}{d z} G_{z}(y)=-\frac{f_{0}(y-2 z)}{2}<0
$$


Using the two equations above yields for (C.12):

$$
\frac{I(c-q \leq y \leq c+q) f_{0}\left(v^{-1}(y)\right)}{2 f_{0}\left(y-2 v^{-1}(y)\right)}-\frac{I(-c-q \leq y \leq-c+q) f_{0}\left(u^{-1}(y)\right)}{2 f_{0}\left(y-2 u^{-1}(y)\right)} .
$$

Noting that $c>q, u^{-1}(y)=-v^{-1}(-y)$, and combining (C.13) with (C.11) gives (4.11).

Derivation of equation (6.3). We show that a necessary condition for $\hat{\boldsymbol{\beta}}$ is (4.2), where $\tilde{\psi}$ is given by (6.4). Then it is clear from Lemma 4 that (6.3) must hold.

Differentiating $\sum_{i=1}^{n} \rho_{1}\left(\frac{H\left(r_{i}(\boldsymbol{\beta})\right)}{s_{n}(\boldsymbol{\beta})}\right)=k_{1}$ yields $\frac{\partial}{\partial \boldsymbol{\beta}} s_{n}(\boldsymbol{\beta})=0$ if

$$
\sum_{i=1}^{n} \psi_{1}\left(\frac{H\left(r_{i}(\boldsymbol{\beta})\right)}{s_{n}(\boldsymbol{\beta})}\right) \frac{\partial}{\partial \boldsymbol{\beta}} H\left(r_{i}(\boldsymbol{\beta})\right)=0
$$

Differentiating the definition of $H\left(r_{i}(\boldsymbol{\beta})\right)$ gives

$$
\frac{\partial}{\partial \boldsymbol{\beta}} H\left(r_{i}(\boldsymbol{\beta})\right)=\frac{\sum_{j=1}^{n} \psi_{2}\left(\frac{r_{i}(\boldsymbol{\beta})-r_{j}(\boldsymbol{\beta})}{H\left(r_{i}(\boldsymbol{\beta})\right)}\right)\left(\mathbf{x}_{j}-\mathbf{x}_{i}\right)}{\sum_{j=1}^{n} \psi_{2}\left(\frac{r_{i}(\boldsymbol{\beta})-r_{j}(\boldsymbol{\beta})}{H\left(r_{i}(\boldsymbol{\beta})\right)}\right) \frac{r_{i}(\boldsymbol{\beta})-r_{j}(\boldsymbol{\beta})}{H\left(r_{i}(\boldsymbol{\beta})\right)}} .
$$

Plugging (C.15) into (C.14), rearranging the indices and noting that $\psi$ is odd yields:

$$
\sum_{i=1}^{n}\left\{\sum_{j=1}^{n} \psi_{2}\left(\frac{r_{i}(\boldsymbol{\beta})-r_{j}(\boldsymbol{\beta})}{H\left(r_{i}(\boldsymbol{\beta})\right)}\right) m(i)+\sum_{j=1}^{n} \psi_{2}\left(\frac{r_{i}(\boldsymbol{\beta})-r_{j}(\boldsymbol{\beta})}{H\left(r_{j}(\boldsymbol{\beta})\right)}\right) m(j)\right\} \mathbf{x}_{i}=0,
$$

where

$$
m(i)=\psi_{1}\left(\frac{H\left(r_{i}(\boldsymbol{\beta})\right)}{s_{n}(\boldsymbol{\beta})}\right) / \sum_{k=1}^{n} \psi_{2}\left(\frac{r_{i}(\boldsymbol{\beta})-r_{k}(\boldsymbol{\beta})}{H\left(r_{i}(\boldsymbol{\beta})\right)}\right) \frac{r_{i}(\boldsymbol{\beta})-r_{k}(\boldsymbol{\beta})}{H\left(r_{i}(\boldsymbol{\beta})\right)} .
$$

From (C.16) it follows that the functional version of the first order necessary condition is given by (4.2).

\section{REFERENCES}

Bradu, D., and Hawkins, D.M. (1993), “Sample Size Requirements for Multiple Outlier Detection Techniques based on Elemental Sets," Computational Statistics and Data Analysis, $16,257-270$.

Carroll, R.J. and Welsh, A.H. (1988), "A Note on Asymmetry and Robustness in Linear Regression," The American Statistician, 42, 285-287. 
Coakley, C.W. and Hettmansperger, T.P. (1993), "A Bounded Influence, High Breakdown, Efficient Regression Estimator," Journal of the American Statistical Association, 88, 872880.

Cook, R.D., Hawkins, D.M., and Weisberg, S. (1992), "Comparison of Model Misspecification Diagnostics Using Residuals From Least Mean of Squares and Least Median of Squares," Journal of the American Statistical Association, 87, 419-424.

Croux, C. (1994), "Efficient High-Breakdown M-Estimators of Scale," Statistics and Probability Letters, 19, 371-379.

Croux, C., and Rousseeuw, P.J. (1992), “Time-Efficient Algorithms for two Highly Robust Estimators of Scale," in Computational Statistics, Volume 1, eds. Y. Dodge and J. Whittaker, Heidelberg: Physika-Verlag, 411-428.

Croux C., and Rousseeuw, P.J. (1994), "High Breakdown Regression by Minimization of a Scale Estimator," in COMPSTAT 1994: Proceedings in Computational Statistics, eds. R. Dutter and W. Grossmann, Heidelberg: Physica-Verlag, 245-250.

Croux, C., Rousseeuw, P.J., and Hössjer, O. (1994), “Generalized S-Estimators," Journal of the American Statistical Association, 89, 1271-1281.

Davies, P.L. (1993), "Aspects of Robust Linear Regression," The Annals of Statistics, 21, $1843-1899$.

Davies, P.L. (1994), "Desirable Properties, Breakdown and Efficiency in the Linear Regression Model," Statistics and Probability Letters, 19, 361-370.

Donoho, D.L., and Huber, P.J. (1983), "The Notion of Breakdown Point," in A Festschrift for Erich Lehmann, eds. P. Bickel, K. Doksum, and J.L. Hodges, Jr., Wadsworth, California.

Hampel, F.R., Ronchetti, E.M., Rousseeuw, P.J., and Stahel, W.A. (1986), Robust Statistics: the Approach based on Influence Functions, New York: John Wiley.

Hawkins, D.M. (1993), “The Feasible Set Algorithm for Least Median of Squares Regression,” Computational Statistics and Data Analysis, 16, 81-101. 
Hawkins, D.M., and Simonoff, J.S. (1993), “AS 282: High Breakdown Regression and Multivariate Estimation," Applied Statistics, 42, 423-432.

He, X., and Simpson, D.G. (1993), "Lower Bounds for Contamination Bias: Globally Minimax Versus Locally Linear Estimation," The Annals of Statistics, 21, 314-337.

Hössjer, O. (1994), "Rank-Based Estimates in the Linear Model with High Breakdown Point," Journal of the American Statistical Association, 89, 149-158.

Martin, R.D., Yohai, V.J., and Zamar, R.H. (1989), "Min-Max Bias Robust Regression," The Annals of Statistics, 17, 1608-1630.

McKean, J.W., Sheather, S.J., and Hettmansperger, T.P. (1993), "The Use and Interpretation of Residuals Based on Robust Estimation," Journal of the American Statistical Association, 88, 1254-1263.

Rousseeuw, P.J. (1983), "Regression Techniques with High Breakdown Point," The IMS Bulletin, 12, 155 .

Rousseeuw, P.J. (1984), "Least Median of Squares Regression," Journal of the American Statistical Association, 79, 871-880.

Rousseeuw, P.J. (1985), "Multivariate Estimation with High Breakdown Point", in Mathematical Statistics and Applications, Vol. B, eds. W. Grossmann, G. Pflug, I. Vincze, and W. Wertz, Dordrecht: Reidel, 283-297.

Rousseeuw, P.J. (1994), “Unconventional Features of Positive-Breakdown Estimators," Statistics and Probability Letters, 19, 417-431.

Rousseeuw, P.J., and Bassett, G.W. (1991), “ Robustness of the p-Subset Algorithm for Regression with High Breakdown Point", in Directions in Robust Statistics and Diagnostics, Part II, eds. W. Stahel and S. Weisberg, New York: Springer Verlag, 185-194.

Rousseeuw, P.J., and Croux, C. (1992), "Explicit Scale Estimators with High Breakdown Point," in $L_{1}$-Statistical Analysis and Related Methods, ed. Y. Dodge, Amsterdam: NorthHolland, 77-92. 
Rousseeuw, P.J., and Croux, C. (1993), "Alternatives to the Median Absolute Deviation," Journal of the American Statistical Association, 88, 1273-1283.

Rousseeuw, P.J., and Leroy, A.M. (1987), Robust Regression and Outlier Detection, New York: John Wiley.

Rousseeuw, P.J., and Yohai, V.J. (1984), "Robust Regression by means of S-estimators," in Robust and Nonlinear Time Series Analysis, eds. J. Franke, W. Härdle and R.D. Martin, Lecture Notes in Statistics 26, New York: Springer Verlag.

Rousseeuw, P.J., and van Zomeren, B.C. (1990), "Unmasking Multivariate Outliers and Leverage Points," Journal of the American Statistical Association, 85, 633-639.

Ruppert, D. (1992), "Computing S-Estimators for Regression and Multivariate Location/Dispersion," Journal of Computational and Graphical Statistics, 1, 253-270.

Sen, P.K. (1968), "Estimates of the Regression Coefficient based on Kendall's Tau," Journal of the American Statistical Association, 63, 1379-1389.

Simpson, D.G., Ruppert, D., and Carroll, R.J. (1992), "On One-Step GM Estimates and Stability of Inferences in Linear Regression," Journal of the American Statistical Association, $87,439-450$.

Tableman, M. (1994), "The Influence Functions for the Least Trimmed Squares and the Least Trimmed Absolute Deviations Estimators," Statistics and Probability Letters, 19 , $329-337$.

Theil, H. (1950), “A Rank-Invariant Method of Linear and Polynomial Regression Analysis (Parts 1-3)," Ned. Akad. Wetensch. Proc. Ser. A., 53, 386-392, 521-525, 1397-1412.

Tichavsky, P. (1991), "Algorithms for and Geometrical Characterization of Solutions in the LMS and the LTS Linear Regression," Computational Statistics Quarterly, 6, 139-151.

Woodruff, D.L., and Rocke, D.M. (1993), "Heuristic Search Algorithms for the Minimum Volume Ellipsoid," Journal of Computational and Graphical Statistics, 2, 69-95.

Yohai, V.J., and Zamar, R.H. (1993), "A Minimax-Bias Property of the Least- $\alpha$-Quantile Estimates," The Annals of Statistics, 21, 1824-1842. 


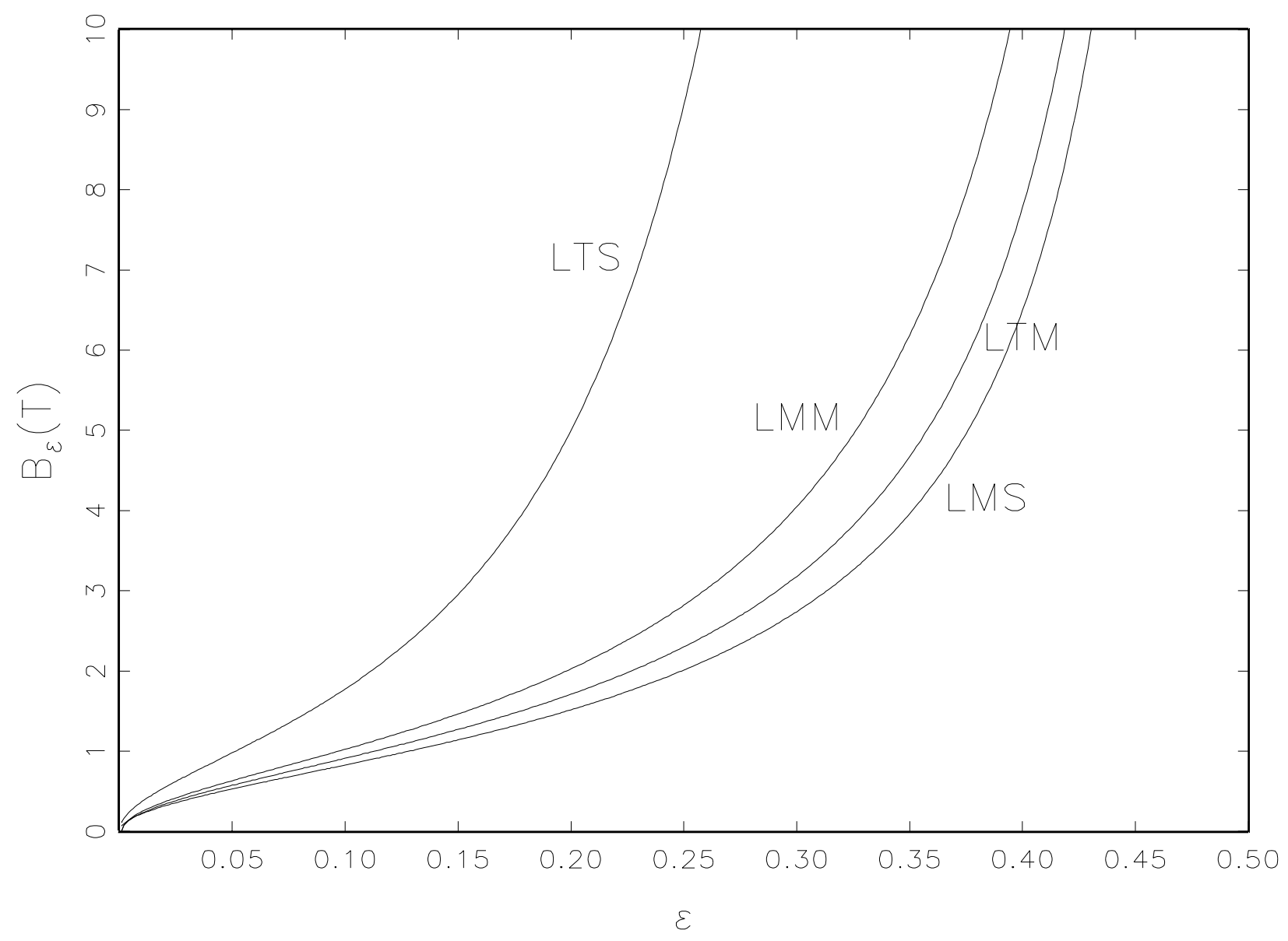

Fig. 1. Maxbias curves of, in increasing order, the LMS, LTM, LMM, and LTS. Lower curves correspond to smaller bias. 


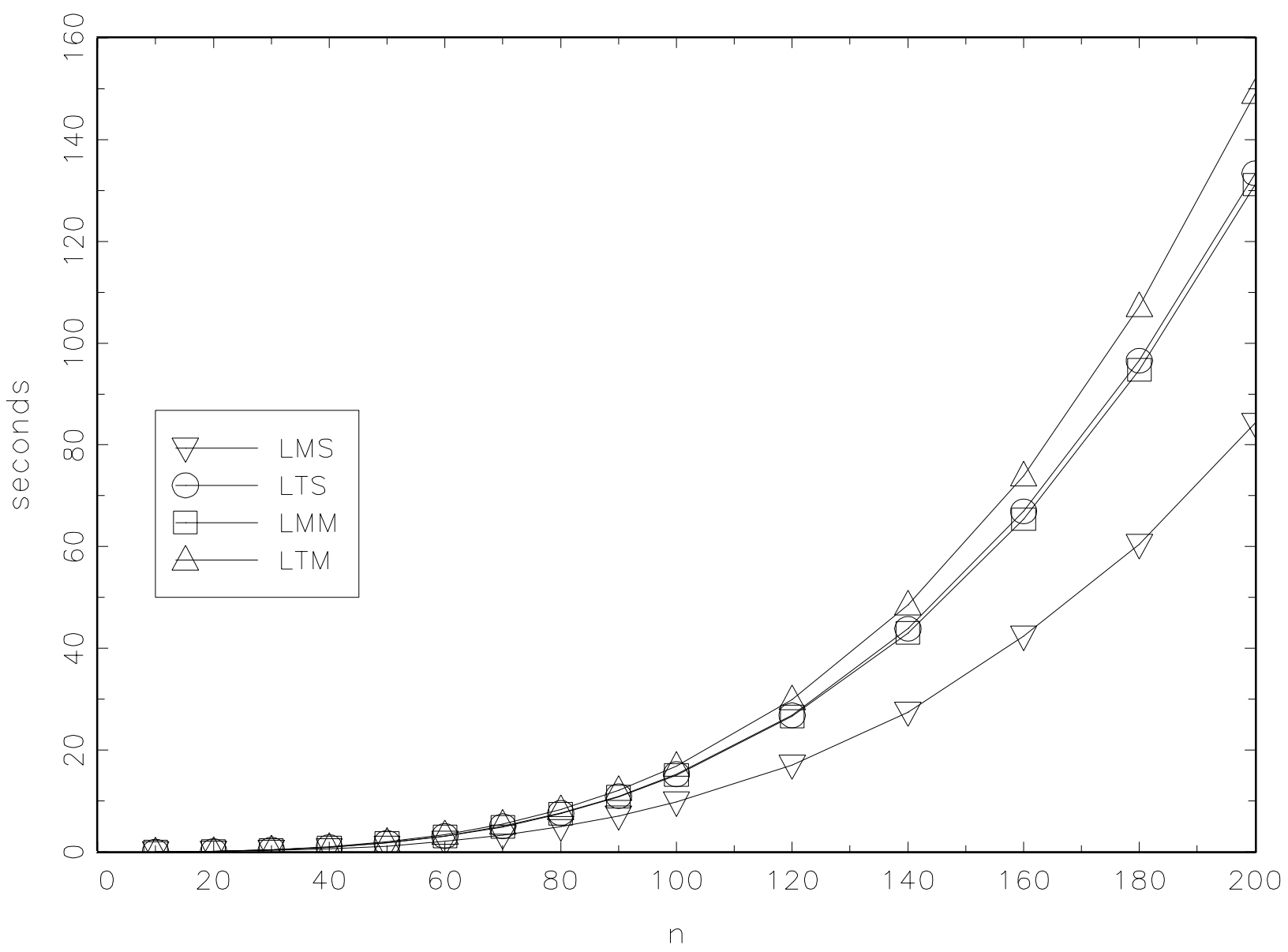

Fig. 2. Computation times of (in increasing order) the LMS, LMM, LTS, and LTM regression estimators (for $p=2$ ). 
(a)

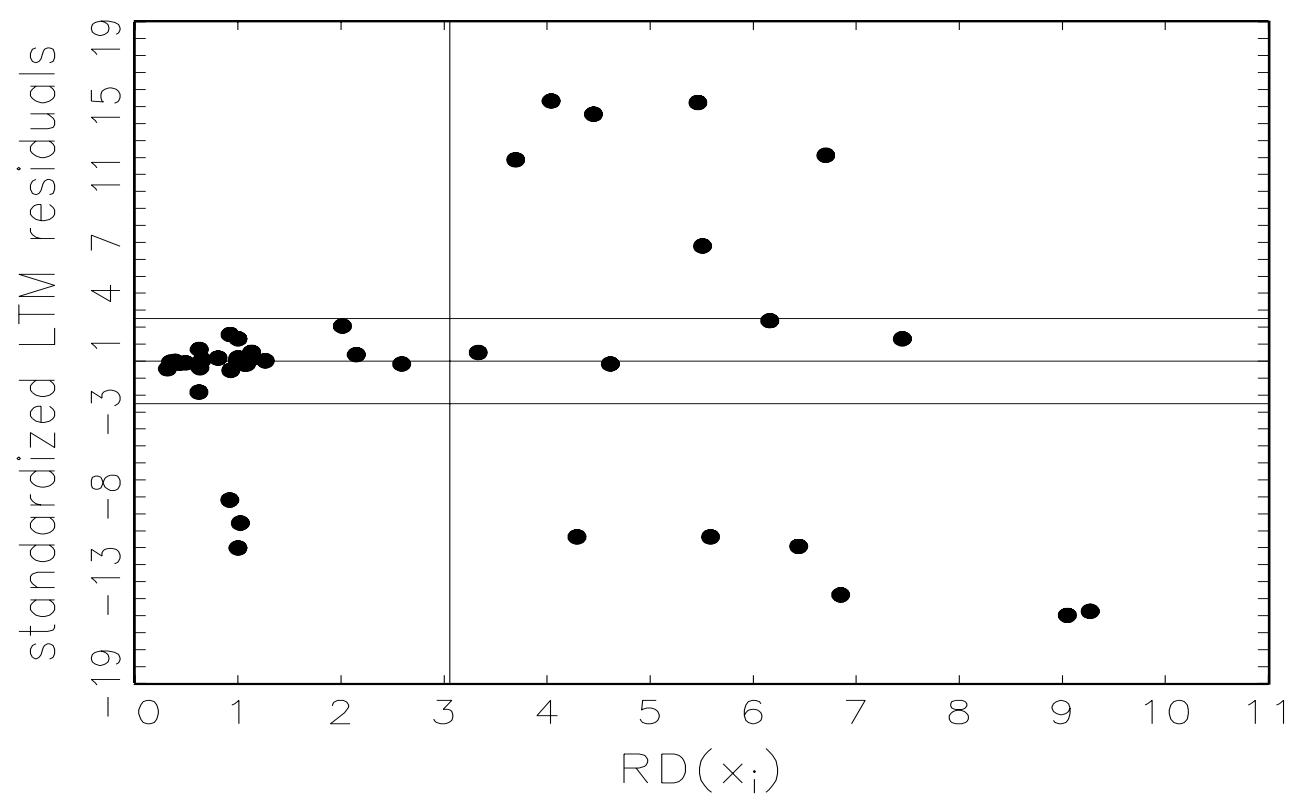

(b)

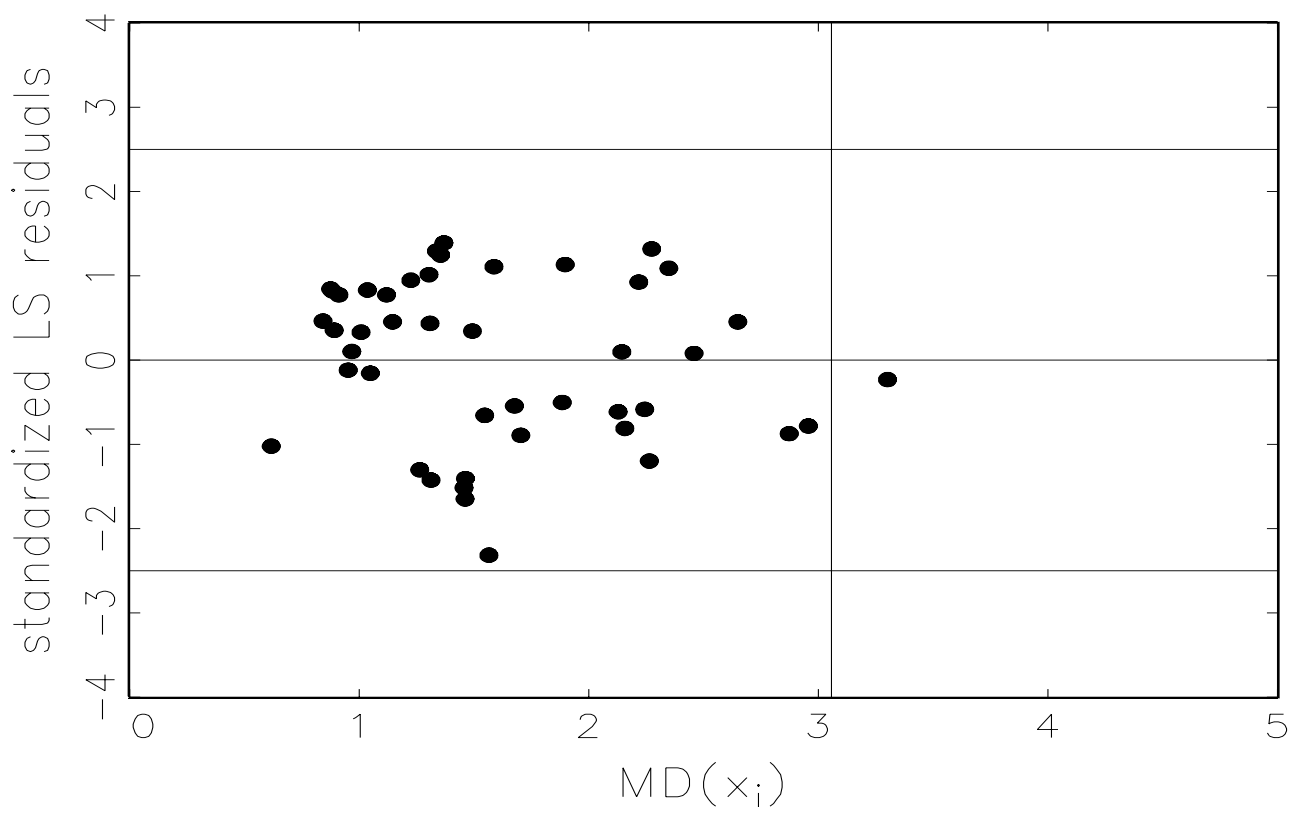

Fig. 3. Plutonium data: (a) standardized LTM residuals $r_{i} / \hat{\sigma}$ versus robust distances $R D\left(\mathbf{x}_{i}\right)$ based on the MVE; (b) standardized LS residuals versus classical Mahalanobis distances $M D\left(\mathrm{x}_{i}\right)$. 

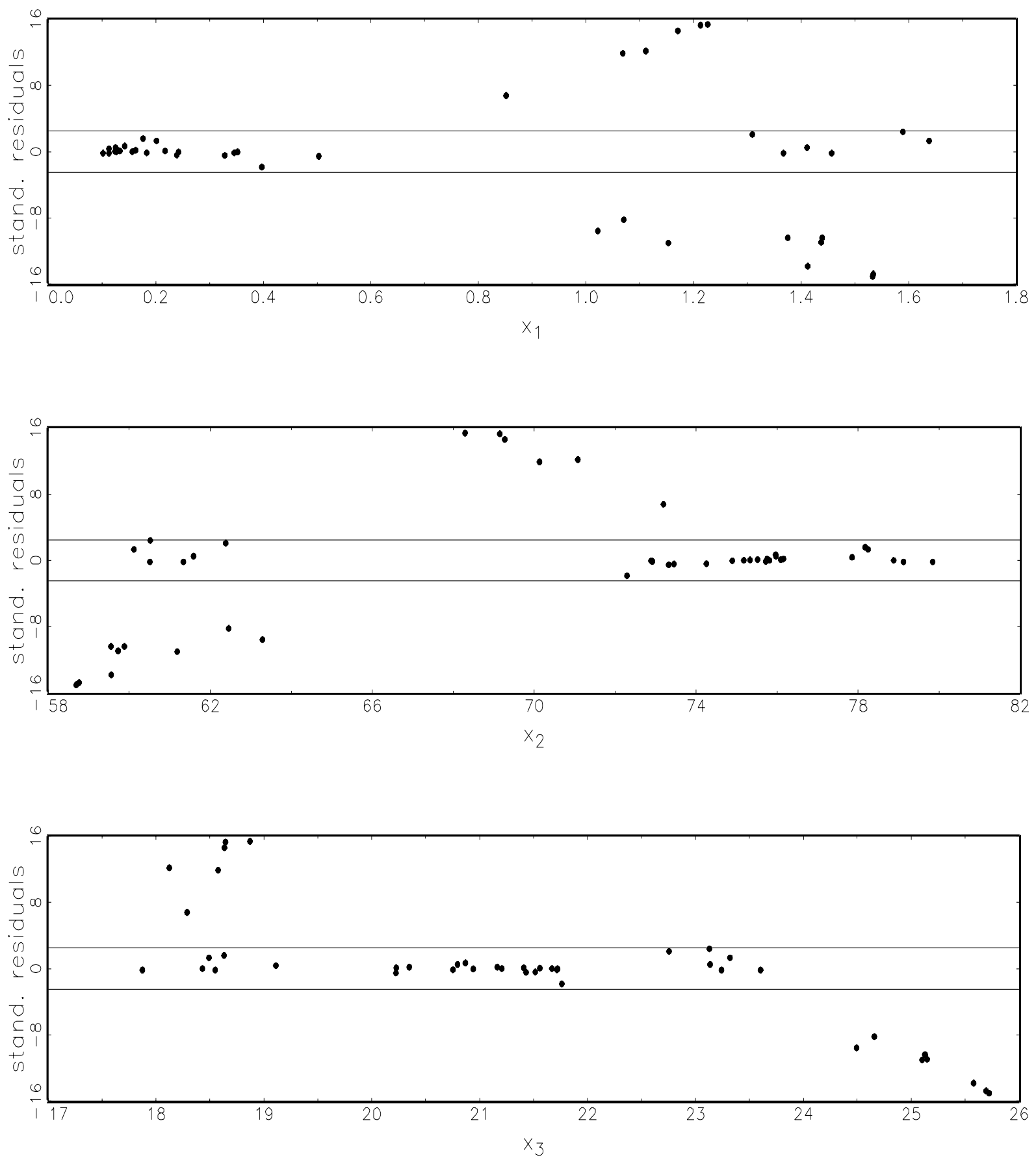

Fig. 3c. Standardized LTM residuals versus the three explanatory variables. 


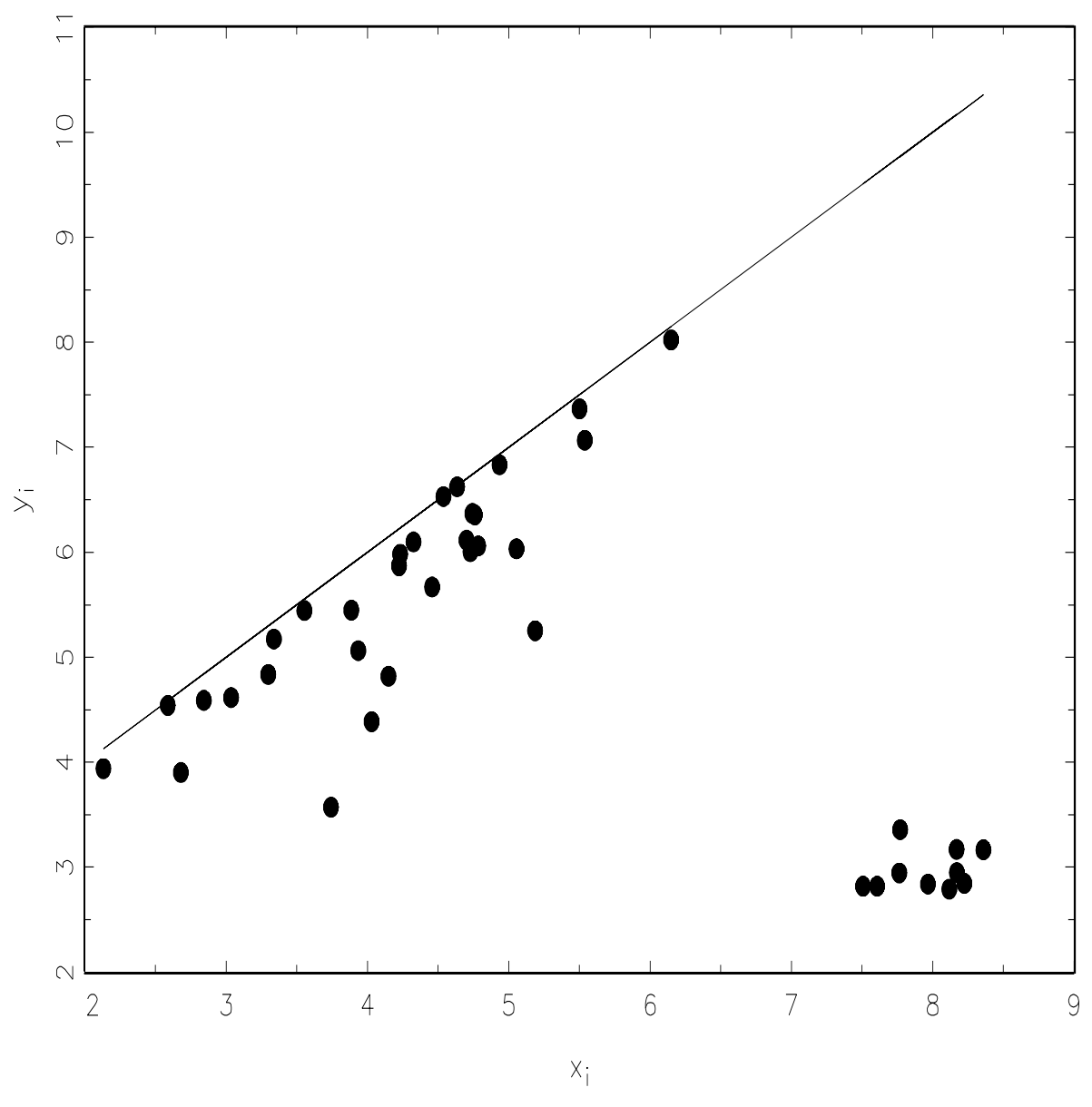

Fig. 4. Simulated data set with 30 observations generated according to $y_{i}=x_{i}+2+\varepsilon_{i}$ where $-2 \varepsilon_{i}$ has the standard exponential distribution, plus 10 outliers. 
(a)

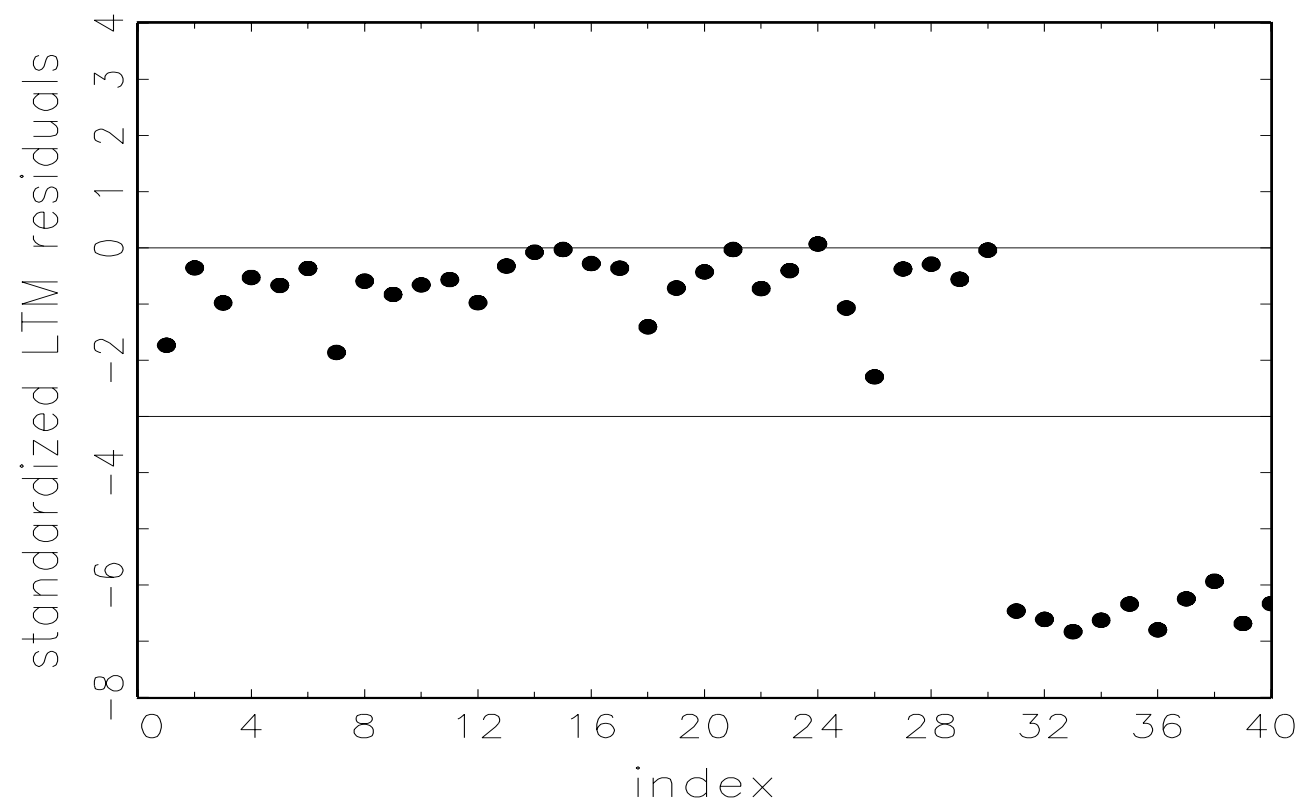

(b)

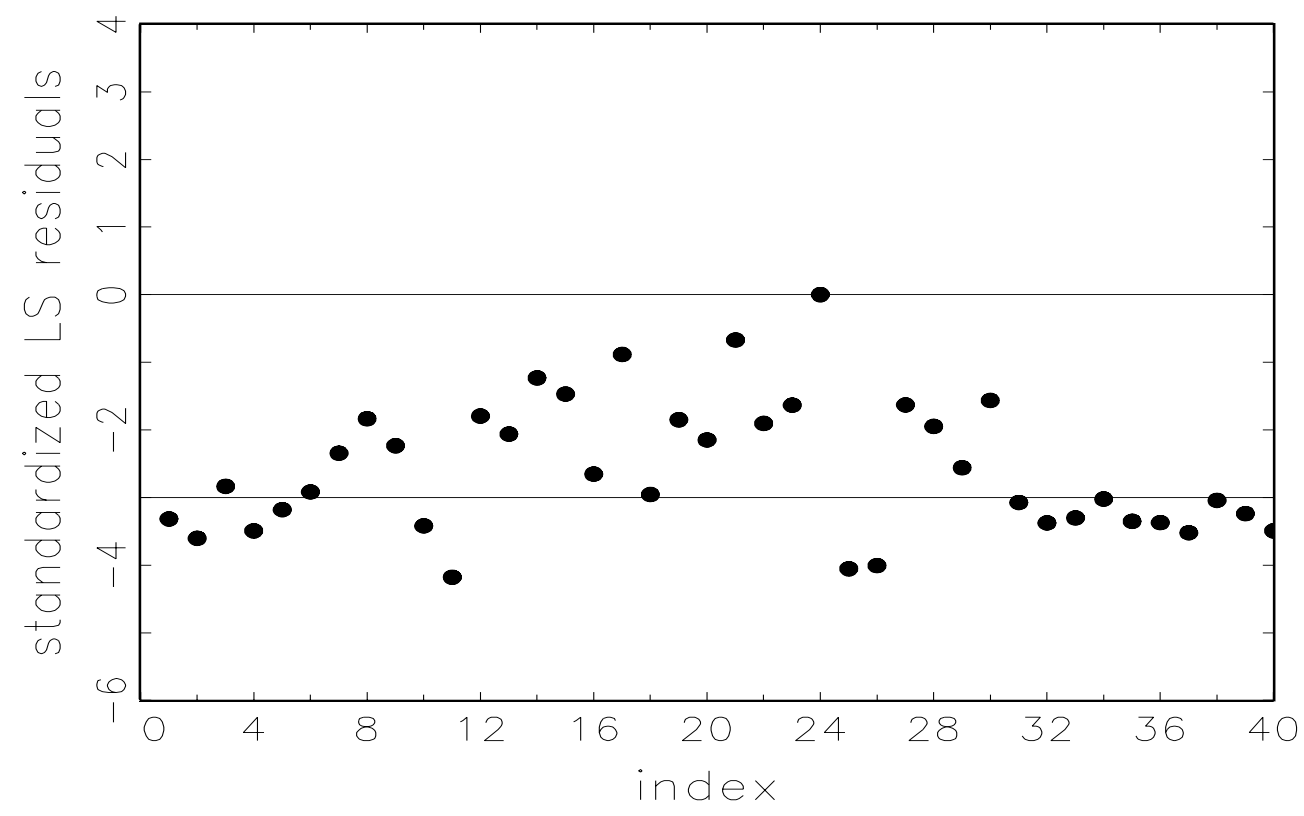

Fig. 5. Residual plots of the data in Figure 4, based on (a) the LTM regression method and (b) the least squares method with MLE intercept. 